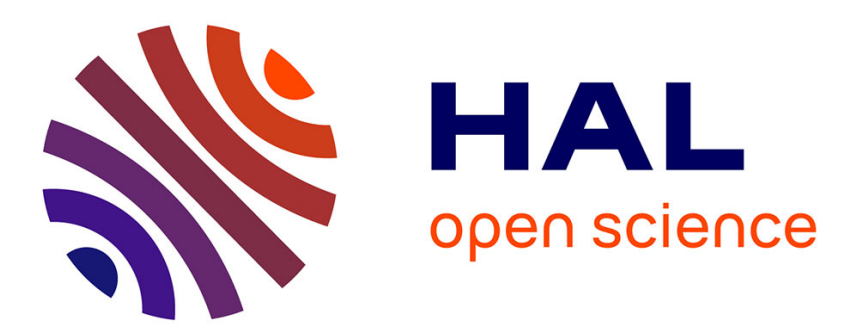

\title{
The Failure of the Strong Pumping Lemma for Multiple Context-Free Languages
}

Makoto Kanazawa, Gregory M. Kobele, Jens Michaelis, Sylvain Salvati, Ryo

Yoshinaka

\section{- To cite this version:}

Makoto Kanazawa, Gregory M. Kobele, Jens Michaelis, Sylvain Salvati, Ryo Yoshinaka. The Failure of the Strong Pumping Lemma for Multiple Context-Free Languages. Theory of Computing Systems, 2014, 55 (1), pp.250-278. 10.1007/s00224-014-9534-z . hal-01251232

\section{HAL Id: hal-01251232 \\ https://hal.science/hal-01251232}

Submitted on 5 Jan 2016

HAL is a multi-disciplinary open access archive for the deposit and dissemination of scientific research documents, whether they are published or not. The documents may come from teaching and research institutions in France or abroad, or from public or private research centers.
L'archive ouverte pluridisciplinaire HAL, est destinée au dépôt et à la diffusion de documents scientifiques de niveau recherche, publiés ou non, émanant des établissements d'enseignement et de recherche français ou étrangers, des laboratoires publics ou privés. 


\title{
The Failure of the Strong Pumping Lemma for Multiple Context-Free Languages
}

\author{
Makoto Kanazawa · Gregory M. Kobele · Jens \\ Michaelis · Sylvain Salvati · Ryo Yoshinaka
}

\begin{abstract}
Seki et al. (Theoretical Computer Science 88(2):191-229, 1991) showed that every $m$-multiple context-free language $L$ is weakly $2 m$-iterative in the sense that either $L$ is finite or $L$ contains a subset of the form $\left\{u_{0} w_{1}^{i} u_{1} \ldots w_{2 m}^{i} u_{2 m} \mid i \in\right.$ $\mathbb{N}\}$, where $w_{1} \ldots w_{2 n} \neq \varepsilon$. Whether every $m$-multiple context-free language $L$ is $2 m$ iterative, that is to say, whether all but finitely many elements $z$ of $L$ can be written as $z=u_{0} w_{1} u_{1} \ldots w_{2 m} u_{2 m}$ with $w_{1} \ldots w_{2 m} \neq \varepsilon$ and $\left\{u_{0} w_{1}^{i} u_{1} \ldots w_{2 m}^{i} u_{2 m} \mid i \in \mathbb{N}\right\} \subseteq L$, has been open. We show that there is a 3-multiple context-free language that is not $k$-iterative for any $k$.
\end{abstract}

Keywords Multiple context-free grammar · Pumping lemma

M. Kanazawa

National Institute of Informatics, 2-1-2 Hitotsubashi, Chiyoda-ku, Tokyo, 101-8430, Japan

E-mail: kanazawa@nii.ac.jp

\section{G.M. Kobele}

Computation Institute and Department of Linguistics, University of Chicago, Chicago, IL 60637, USA

E-mail: kobele@uchicago.edu

J. Michaelis

Fakultät für Linguistik und Literaturwissenschaft, Universität Bielefeld, Postfach 1001 31, D-33501 Bielefeld, Germany

E-mail: jens.michaelis@uni-bielefeld.de

S. Salvati

INRIA Bordeaux Sud-Ouest, LaBRI, 351, cours de la Libération, F-33405 Talence cedex, France

E-mail: sylvain.salvati@labri.fr

R. Yoshinaka

Graduate School of Informatics, Kyoto University, 36-1 Yoshida-Honmachi, Sakyo-ku, Kyoto, 606-8501,

Japan

E-mail: ry@i.kyoto-u.ac.jp 


\section{Introduction}

The study of iterative properties of the languages of multiple context-free grammars (MCFG) [14] has had a peculiar history [1] Seki et al. [14] proved that any language $L$ generated by an MCFG of dimension $m$ (i.e., $m$-MCFG) is weakly $2 m$-iterative (in the sense of Greibach [3,2]): either $L$ is finite or else it contains a subset of the form

$$
\left\{u_{0} w_{1}^{i} u_{1} \ldots w_{2 m}^{i} u_{2 m} \mid i \in \mathbb{N}\right\}
$$

for some strings $u_{0}, u_{1}, \ldots, u_{2 m}$ and $w_{1}, \ldots, w_{2 m}$ such that $w_{1} \ldots w_{2 m} \neq\left.\varepsilon\right|^{2}$ Seki et al. [14] called this theorem a "pumping lemma" for $m$-MCFLs. Their proof of the theorem starts with an application of the pigeon-hole principle to a path in a derivation tree in a way familiar from the pumping lemma for context-free languages; beyond that, however, it involves much more intricate reasoning than in the context-free case, due to the complex relation between derivation trees of an MCFG and the derived strings. The proof goes roughly as follows.

Given a sufficiently long string $z$ in the language $L$ of an $m$-MCFG $G$, the derivation tree $T$ for $z$ must contain a "context" $U[]$ inside it that can be iterated any number of times $3^{3}$ That is to say, $T$ can be written as $T=U^{\prime}\left[U\left[T^{\prime}\right]\right]$, where $U\left[T^{\prime}\right]$ is a subtree of $T$ which contains $T^{\prime}$ as a proper subtree, and for each $i \geq 0, U^{\prime}\left[U^{i}\left[T^{\prime}\right]\right]$ is also a derivation tree. Here, the notation $U^{i}\left[T^{\prime}\right]$ is defined by

$$
\begin{aligned}
U^{0}\left[T^{\prime}\right] & =T^{\prime}, \\
U^{i+1}\left[T^{\prime}\right] & =U\left[U^{i}\left[T^{\prime}\right]\right] .
\end{aligned}
$$

In the case of a context-free grammar, each subtree of a derivation tree yields a single string. In the case of an $m$-MCFG, in contrast, each subtree of a derivation tree is associated with a tuple of strings. Thus, the contribution of the iterable context $U[]$ to the derived string is some function $g$ mapping an $n$-tuple of strings to another $n$-tuple, for some $n \leq m$. Such a function can be specified by an equation of the form $g\left(\boldsymbol{x}_{1}, \ldots, \boldsymbol{x}_{n}\right)=\left(\alpha_{1}, \ldots, \alpha_{n}\right)$ using variables $\boldsymbol{x}_{i}$ and strings $\alpha_{i}$ over $\Sigma \cup\left\{\boldsymbol{x}_{1}, \ldots, \boldsymbol{x}_{n}\right\}$, where $\Sigma$ is the terminal alphabet, such that each $\boldsymbol{x}_{i}$ occurs in a unique $\alpha_{j}$. In the special case where $\alpha_{j}=w_{2 j-1} \boldsymbol{x}_{j} w_{2 j}$ for all $j=1, \ldots, n$ $\left(w_{1}, \ldots, w_{2 n} \in \Sigma^{*}\right)$, iteration of $U[]$ inside the derivation tree translates into iteration of the strings $w_{1}, \ldots, w_{2 n}$ inside the derived string, giving rise to a set of the form (1). In general, since $\boldsymbol{x}_{i}$ may end up in some $\alpha_{j}$ with $j \neq i$, the effect of iterating $U]$ in $T=U^{\prime}\left[U\left[T^{\prime}\right]\right]$ is rather hard to describe. As a consequence, derivation trees of the form $U^{\prime}\left[U^{i}\left[T^{\prime}\right]\right]$ do not (necessarily) generate a set of the form (1). One can see, however, that for large enough $k$, the $k$-fold composition $g^{k}$ of $g$ with itself has the property that if $g^{k}\left(\boldsymbol{x}_{1}, \ldots, \boldsymbol{x}_{n}\right)=\left(\beta_{1}, \ldots, \beta_{n}\right)$, then for every $j=1, \ldots, n$,

\footnotetext{
${ }^{1}$ Around the same time as Kasami et al. [9] first introduced multiple context-free grammars, essentially the same formalism was proposed by Vijay-Shanker et al. [15] under the name linear context-free rewriting systems (LCFRS). In this paper, we mostly follow the terminology of Seki et al. [14].

2 We let $\mathbb{N}$ denote the set of natural numbers $\{0,1,2, \ldots\}$ and $\varepsilon$ denote the empty string.

${ }^{3}$ Formally, a context is a tree with a single special leaf node ("hole"), which is labeled by $\square$. When $U[]$ is a context and $T$ is a tree, $U[T]$ denotes the tree that results from removing the hole of $U[]$ and inserting $T$ in its place.
} 
$\beta_{j}$ either is a constant string (i.e., string over $\Sigma$ ) or else contains $\boldsymbol{x}_{j}$. It follows that $g^{2 k}\left(\boldsymbol{x}_{1}, \ldots, \boldsymbol{x}_{n}\right)=g^{k}\left(\beta_{1}, \ldots, \beta_{n}\right)=\left(w_{1} \beta_{1} w_{2}, \ldots, w_{2 n-1} \beta_{n} w_{2 n}\right)$ for some constant strings $w_{1}, \ldots, w_{2 n}$ such that $w_{2 j-1} w_{2 j}=\varepsilon$ whenever $\beta_{j}$ is a constant string. It is not difficult to see that this implies that $g^{(i+1) k}\left(\boldsymbol{x}_{1}, \ldots, \boldsymbol{x}_{n}\right)=\left(w_{1}^{i} \beta_{1} w_{2}^{i}, \ldots, w_{2 n-1}^{i} \beta_{n} w_{2 n}^{i}\right)$. Thus, derivation trees $U^{\prime}\left[U^{(i+1) k}\left[T^{\prime}\right]\right](i \geq 0)$ yield a subset of $L$ of the required form (1). Crucially, the original string $z$ is not an element of this set.

By a strange quirk of fate, this proof was erroneously claimed by Radzinski [13] to implicitly demonstrate a much stronger property ${ }^{4}$ namely, that every $m$-MCFL $L$ is $2 m$-iterative (in the sense of Greibach [3]): all but finitely many $z \in L$ can be written as $z=u_{0} w_{1} u_{1} \ldots w_{2 m} u_{2 m}$ such that $w_{1} \ldots w_{2 m} \neq \varepsilon$ and $\left\{u_{0} w_{1}^{i} u_{1} \ldots w_{2 m}^{i} u_{2 m} \mid i \in \mathbb{N}\right\} \subseteq L$. More strangely, Groenink [5] just took Radzinski's word for it (see also [4]). A more recent book by Kracht [10] also states this property as a theorem.

We refer to the assertion that every $m$-MCFL is $2 m$-iterative as the strong pumping lemma for $m$-MCFLs, to distinguish it from Seki et al.'s [14] theorem. It is clear that no simple modification of the method of Seki et al. can establish the strong pumping lemma for $m$-MCFLs. It is only when the iterable context $U$ [] maps an $n$-tuple $\left(\boldsymbol{x}_{1}, \ldots, \boldsymbol{x}_{n}\right)$ to an $n$-tuple of the form $\left(w_{1} \boldsymbol{x}_{1} w_{2}, \ldots, w_{2 n-1} \boldsymbol{x}_{n} w_{2 n}\right)$ that it is possible to conclude, analogously to the context-free case, that the given string $z$ contains factors $w_{1}, \ldots, w_{2 m}$ that can be pumped up and down without pushing the resulting string outside of the given $m$-MCFL $[5$ Kanazawa [6] called such a well-behaved iterable context an even pump in his proof that an $m$-MCFG satisfying the condition of wellnestedness always generates a $2 m$-iterative set. This proof works by induction on $m$. The base case is handled by the fact that well-nested 1-MCFGs are just CFGs. For the induction step, Kanazawa showed that given a well-nested $m$-MCFG $G$, one can always find a well-nested $(m-1)$-MCFG $G^{\prime}$ for the language $L^{\prime}$ consisting of strings generated by $G$ with derivation trees containing no even pump. Hence the language $L$ of $G$ is a union of some $2 m$-iterative set and $L^{\prime}$, which, by induction hypothesis, is a $2(m-1)$-iterative set. It follows that $L$ is $2 m$-iterative, completing the induction. This method is such that derivation trees of $G^{\prime}$ have very different shapes from the original derivation trees of $G$ for the same strings. Whereas the method also works for 2-MCFGs in general, the well-nestedness property is essential for $m \geq 3$, and there is no obvious way of extending it to the non-well-nested case.

In this paper, we prove that the strong pumping lemma indeed fails for non-wellnested $m$-MCFGs for $m \geq 3$. We do so by exhibiting a particular 3-MCFG that generates a language that is non-iterative in a very strong sense. This language, which we call $H$, is not $k$-iterative for any $k$. It is not even finitely pumpable in the sense of Groenink [5, 4], a condition which is similar to $k$-iterativity but allows the number of iterable factors to vary from string to string. In fact, $H$ contains an infinite subset $\left\{v_{n} \mid n \in \mathbb{N}\right\}$ consisting of strings that are almost anti-iterative in the following sense: whenever $v_{n}=u_{0} w_{1} u_{1} \ldots w_{k} u_{k}$ and $w_{1} \ldots w_{k} \neq \varepsilon$ (for any $k$ ), it holds that

$$
\mid\left\{i \mid i>1 \text { and } u_{0} w_{1}^{i} u_{1} \ldots w_{k}^{i} u_{k} \in H\right\} \mid \leq 1
$$

${ }^{4}$ See footnote 10 of Radzinski [13]. Radzinski refers to the technical report [9] rather than the journal article [14] based on it, but the proof is the same in both papers.

${ }^{5}$ A string $v$ is a factor of a string $z$ if $z=u v w$ for some strings $u, w$. 
Most of the rest of the paper is devoted to the proof of this property of the language $H$ (section 3). Before we get to it, we briefly review basic notions concerning multiple context-free grammars for readers unfamiliar with this grammar formalism (section 2). The proof in section 3 does not use any general properties of MCFLs, and can be followed by anyone who understands the definition of the language $H$.

\section{Multiple Context-Free Grammars}

Like a context-free grammar, a multiple context-free grammar is a quadruple $G=$ $(N, \Sigma, P, S)$, where $N$ is a finite set of nonterminals, $\Sigma$ is a finite set of terminals, $P$ is a set of rules, and $S$ is a designated nonterminal. While a nonterminal of a CFG is associated with a set of terminal strings, a nonterminal of an MCFG is interpreted as a $q$-ary relation on terminal strings, where $q$ is the dimension of the nonterminal. Each nonterminal comes with a unique dimension. (So the set $N$ can be thought of as a ranked alphabet.) The dimension of the designated nonterminal $S$ is always 1 . A rule is of the form

$$
A\left(\alpha_{1}, \ldots, \alpha_{q}\right) \leftarrow B_{1}\left(\boldsymbol{x}_{1,1}, \ldots, \boldsymbol{x}_{1, q_{1}}\right), \ldots, B_{n}\left(\boldsymbol{x}_{n, 1}, \ldots, \boldsymbol{x}_{n, q_{n}}\right),
$$

where $n \geq 0, A, B_{1}, \ldots, B_{n}$ are nonterminals of dimension $q, q_{1}, \ldots, q_{n}$, respectively, the $\boldsymbol{x}_{i, j}$ are pairwise distinct variables, which are symbols not in $\Sigma$, and $\alpha_{1}, \ldots, \alpha_{q}$ are strings over $\Sigma \cup\left\{\boldsymbol{x}_{i, j} \mid 1 \leq i \leq n, 1 \leq j \leq q_{i}\right\}$ such that each $\boldsymbol{x}_{i, j}$ occurs at most once in $\alpha_{1} \ldots \alpha_{q}$.

A rule is interpreted like a universally quantified implication from right to left. Define a predicate $\vdash_{G}$ that holds of expressions of the form $A\left(u_{1}, \ldots, u_{q}\right)$ (called facts) inductively as follows:

- If $A\left(u_{1}, \ldots, u_{q}\right) \leftarrow$ is a rule of $G$, then $\vdash_{G} A\left(u_{1}, \ldots, u_{q}\right)$.

- If $A\left(\alpha_{1}, \ldots, \alpha_{q}\right) \leftarrow B_{1}\left(\boldsymbol{x}_{1,1}, \ldots, \boldsymbol{x}_{1, q_{1}}\right), \ldots, B_{n}\left(\boldsymbol{x}_{n, 1}, \ldots, \boldsymbol{x}_{n, q_{n}}\right)$ is a rule of $G$ and $\vdash_{G}$ $B_{i}\left(w_{i, 1}, \ldots, w_{i, q_{i}}\right)$ for $i=1, \ldots, n$, then $\vdash_{G} A\left(u_{1}, \ldots, u_{q}\right)$, where $\left(u_{1}, \ldots, u_{q}\right)$ is the result of substituting $w_{i, j}$ for each $\boldsymbol{x}_{i, j}$ in $\left(\alpha_{1}, \ldots, \alpha_{q}\right)$.

When $\vdash_{G} A\left(u_{1}, \ldots, u_{q}\right)$, we say that $A\left(u_{1}, \ldots, u_{q}\right)$ is derivable (in $G$ ). (We sometimes write $\vdash$ instead of $\vdash_{G}$ when the grammar is clear from the context.) The language of $G$ is defined by $L(G)=\left\{w \in \Sigma^{*} \mid \vdash_{G} S(w)\right\}$.

An MCFG is an $m-M C F G$ if the dimension of nonterminals does not exceed $m$. The language of an $m$-MCFG is called an $m-M C F L$. It is shown by Seki et al. [14] that each $m$-MCFG has an equivalent one such that the variables on the right-hand side of any rule all appear in the left-hand side. Such an MCFG is called non-deleting.

A rule $A\left(\alpha_{1}, \ldots, \alpha_{q}\right) \leftarrow B_{1}\left(\boldsymbol{x}_{1,1}, \ldots, \boldsymbol{x}_{1, q_{1}}\right), \ldots, B_{n}\left(\boldsymbol{x}_{n, 1}, \ldots, \boldsymbol{x}_{n, q_{n}}\right)$ is called nonpermuting if for each $i=1, \ldots, n$ and each $j, k$ such that $1 \leq j<k \leq q_{i}$, it is not the case that

$$
\varphi\left(\alpha_{1} \ldots \alpha_{q}\right)=\boldsymbol{x}_{i, k} \boldsymbol{x}_{i, j},
$$

where $\varphi$ is the homomorphism that erases all symbols in $\Sigma$ and all variables other than $\boldsymbol{x}_{i, j}$ and $\boldsymbol{x}_{i, k}$. An MCFG $G$ is called non-permuting if all its rules are non-permuting. Every $m$-MCFG has an equivalent non-deleting non-permuting $m$-MCFG [11,10]. 
A non-deleting non-permuting MCFG is called well-nested if every rule $A\left(\alpha_{1}, \ldots, \alpha_{q}\right) \leftarrow B_{1}\left(x_{1,1}, \ldots, x_{1, q_{1}}\right), \ldots, B_{n}\left(\boldsymbol{x}_{n, q}, \ldots, \boldsymbol{x}_{n, q_{n}}\right)$ satisfies the following condition: whenever $i \neq i^{\prime}, 1 \leq j<k \leq q_{i}, 1 \leq j^{\prime}<k^{\prime} \leq q_{i^{\prime}}$, it is not the case that

$$
\chi\left(\alpha_{1} \ldots \alpha_{q}\right)=\boldsymbol{x}_{i, j} \boldsymbol{x}_{i^{\prime}, j^{\prime}} \boldsymbol{x}_{i, k} \boldsymbol{x}_{i^{\prime}, k^{\prime}}
$$

where $\chi$ is the homomorphism that erases all symbols in $\Sigma$ and all variables other than $\boldsymbol{x}_{i, j}, \boldsymbol{x}_{i, k}, \boldsymbol{x}_{i^{\prime}, j^{\prime}}, \boldsymbol{x}_{i^{\prime}, k^{\prime}}$. Kanazawa [6] showed that the languages of well-nested $\mathrm{m}$ MCFGs are all $2 m$-iterative. See also [8] for the effect of the well-nestedness condition on the generative power of MCFGs.

In order to rigorously define the notion of a derivation tree, we view the rule set $P$ as a ranked alphabet where $\pi \in P$ has rank $n$ if the right-hand side of $\pi$ has $n$ occurrences of nonterminals. A derivation tree of $G=(N, \Sigma, P, S)$ is a local set of trees over $P$, defined inductively as follows:

- If $\pi=A\left(u_{1}, \ldots, u_{q}\right) \leftarrow$ is a rule in $P$, then $\pi$ is a derivation tree for $A\left(u_{1}, \ldots, u_{q}\right)$.

- If $\pi=A\left(\alpha_{1}, \ldots, \alpha_{q}\right) \leftarrow B_{1}\left(\boldsymbol{x}_{1,1}, \ldots, \boldsymbol{x}_{1, q_{1}}\right), \ldots, B_{n}\left(\boldsymbol{x}_{n, 1}, \ldots, \boldsymbol{x}_{n, q_{n}}\right)$ is a rule in $P$ and for $i=1, \ldots, n, T_{i}$ is a derivation tree for $B_{i}\left(w_{i, 1}, \ldots, w_{i, q_{i}}\right)$, then $\pi T_{1} \ldots T_{n}$ is a derivation tree for $A\left(u_{1}, \ldots, u_{q}\right)$, where $\left(u_{1}, \ldots, u_{q}\right)$ is the result of substituting $w_{i, j}$ for each $\boldsymbol{x}_{i, j}$ in $\left(\alpha_{1}, \ldots, \alpha_{q}\right)$.

A derivation tree for $A\left(u_{1}, \ldots, u_{q}\right)$ is a derivation tree of type A. A complete derivation tree is a derivation tree of type $S$, and it is said to be a derivation tree for $w$ if it is a derivation tree for $S(w)$. When $T$ is a derivation tree for a fact $A\left(u_{1}, \ldots, u_{q}\right)$, we also say $T$ derives $A\left(u_{1}, \ldots, u_{q}\right)$. Clearly, $\vdash_{G} A\left(u_{1}, \ldots, u_{q}\right)$ holds if and only if $G$ has a derivation tree that derives $A\left(u_{1}, \ldots, u_{q}\right)$.

When a derivation tree of type $B$ contains a derivation tree of type $A$ as a subtree, the result of replacing that subtree by any other derivation tree of type $A$ is again a derivation tree of type $B$. When a complete derivation tree $T$ for $w$ has a path containing more nodes than the number of nonterminals, then there must be a nonterminal $A$ and two nodes on that path such that the subtree rooted at each of the two nodes is a derivation tree of type $A$. This is the starting point of Seki et al.'s [14] proof of their pumping lemma.

Example 1 Consider the following 2-MCFG:

$$
\begin{aligned}
& \pi_{1}: S\left(x_{1} \# x_{2}\right) \leftarrow D\left(x_{1}, x_{2}\right) \\
& \pi_{2}: D(\varepsilon, \varepsilon) \leftarrow \\
& \pi_{3}: D\left(x_{1} y_{1}, x_{2} y_{2}\right) \leftarrow E\left(x_{1}, x_{2}\right), D\left(y_{1}, y_{2}\right) \\
& \pi_{4}: E\left(c x_{1} \bar{c}, c x_{2} \bar{c}\right) \leftarrow D\left(x_{1}, x_{2}\right)
\end{aligned}
$$

$\left(\pi_{1}, \pi_{2}, \pi_{3}, \pi_{4}\right.$ are the names of the rules.) Here, $S$ is the designated nonterminal, and all other nonterminals are of rank 2. This grammar generates $\left\{w \# w \mid w \in D_{1}^{*}\right\}$, where $D_{1}^{*}$ is the Dyck language over the alphabet $\{c, \bar{c}\}$. Note that the third rule is not wellnested. Figure 1 shows a derivation tree for $c c \bar{c} \bar{c} c \bar{c} \# c c \bar{c} \bar{c} c \bar{c}$, alongside of the same tree with each node annotated by the fact derived by the subtree rooted at that node. 

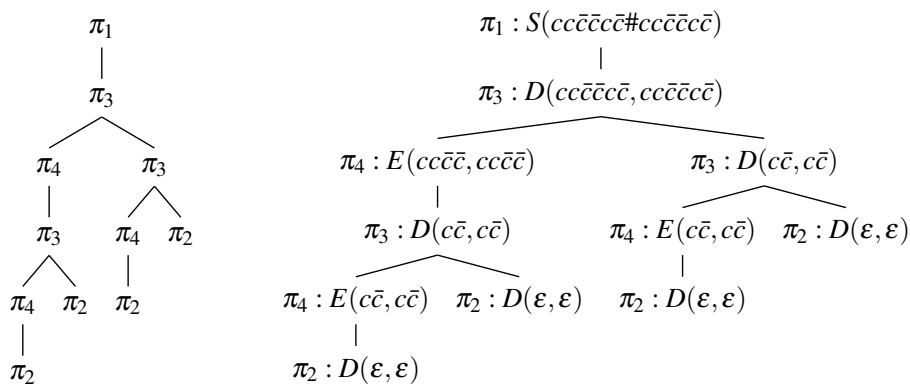

Fig. 1 A derivation tree for $c c \bar{c} \bar{c} c \bar{c} \# c c \bar{c} \bar{c} c \bar{c}$ (left) and the same tree augmented with additional information about what fact is derived at each step (right).

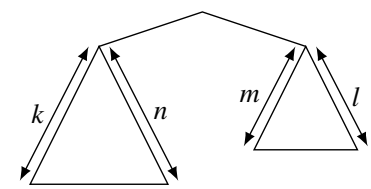

Fig. 2 Derivation tree for $J\left(a^{k+1}, a^{m} c v \bar{c} d w \bar{d} b^{n}, b^{l+1}\right)$.

\section{Counterexample to the Strong Pumping Lemma for 3-MCFLs}

We fix two alphabets:

$$
\begin{aligned}
& \Sigma=\{c, \bar{c}, d, \bar{d}\}, \\
& \hat{\Sigma}=\Sigma \cup\{a, b\} .
\end{aligned}
$$

Define a 3-MCFL $H \subseteq \hat{\Sigma}^{*}$ by the following 3-MCFG, where we use the symbol $H$ itself as the designated nonterminal:

$$
\begin{aligned}
& H\left(x_{2}\right) \leftarrow J\left(x_{1}, x_{2}, x_{3}\right) \\
& J\left(a x_{1}, y_{1} c x_{2} \bar{c} d y_{2} \bar{d} x_{3}, y_{3} b\right) \leftarrow J\left(x_{1}, x_{2}, x_{3}\right), J\left(y_{1}, y_{2}, y_{3}\right) \\
& J(a, \varepsilon, b) \leftarrow
\end{aligned}
$$

This is our counterexample to the strong pumping lemma. Note that the second rule is not well-nested. When $J\left(u_{1}, u_{2}, u_{3}\right)$ is derivable in this grammar, we always have $u_{1}=a^{k+1}, u_{3}=b^{l+1}$ for some $k, l \in \mathbb{N}$, and $u_{2}$ is either $\varepsilon$ or a string of the form $a^{m} c v \bar{c} d w \bar{d} b^{n}$ for some $v, w \in H$ and $m, n \geq 1$. In the latter case, the (unique) derivation tree for $J\left(a^{k+1}, a^{m} c v \bar{c} d w \bar{d} b^{n}, b^{l+1}\right)$ is a binary tree $T$ where $k$ and $n$ are the numbers of nodes on the leftmost and rightmost branches, respectively, of the left immediate subtree of $T$, and $m$ and $l$ are the numbers of nodes on the leftmost and rightmost branches, respectively, of the right immediate subtree of $T$ (Figure 2).

The language $H$ is related to a context-free language over $\Sigma$ via the homomorphism $\psi: \hat{\Sigma}^{*} \rightarrow \Sigma^{*}$ defined by:

$$
\psi(e)= \begin{cases}\varepsilon & \text { if } e \in\{a, b\} \\ e & \text { if } e \in \Sigma\end{cases}
$$


It is easy to see that $\psi(H)$ is a context-free language included in the Dyck language $D_{2}^{*}$ over the alphabet $\Sigma$, where $(c, \bar{c})$ and $(d, \bar{d})$ are each regarded as a matching pair of parentheses. The homomorphism $\psi$ is an injection when restricted to the strings in $H$, and for each $v \in H, \psi(v)$ encodes in an obvious way the unique derivation tree for $v$. We can learn a lot about iterative properties of the 3-MCFL $H$ from the CFL $\psi(H)$, so we begin by studying the latter.

\subsection{Properties of the CFL $V=\psi(H)$}

The goal of this section is to state a necessary condition for $w \in \Sigma^{+}$to be in

$$
\{w \mid w w \text { is a factor of some string in } \psi(H)\} \text {. }
$$

In what follows, we use regular expressions and (recursive) equations involving regular expressions to define various languages. In regular expressions, the vertical bar "|" denotes union, and is assumed to have lower precedence than all other operators.

Define the reduction relation $\triangleright \in \Sigma^{*} \times \Sigma^{*}$ by

$$
\triangleright=\left\{\left(v_{1} c \bar{c} v_{2}, v_{1} v_{2}\right) \mid v_{1}, v_{2} \in \Sigma^{*}\right\} \cup\left\{\left(v_{1} d \bar{d} v_{2}, v_{1} v_{2}\right) \mid v_{1}, v_{2} \in \Sigma^{*}\right\} .
$$

We write $\triangleright^{*}$ for the reflexive transitive closure of the relation $\triangleright$, and $\triangleright^{n}$ for the $n$-fold composition of $\triangleright$ with itself (more precisely, $\triangleright^{n+1}$ is $\triangleright$ composed with $\triangleright^{n}$, where $\triangleright^{0}$ is the identity relation). When $v \triangleright^{*} w$, we say $v$ reduces to $w$, and when $v \triangleright^{n} w$, we say $v$ reduces to $w$ in $n$ steps. A string $w \in \Sigma^{*}$ is said to be in normal form if neither $c \bar{c}$ nor $d \bar{d}$ is a factor of $w$. It is well known that the relation $\triangleright^{*}$ has the confluence (i.e., Church-Rosser) property and each string $w \in \Sigma^{*}$ reduces to a unique string in normal form, which is called the normal form of $w$. We write $\mathrm{nf}(w)$ for the normal form of $w$. The Dyck language $D_{2}^{*}$ over $\Sigma$ is defined as $D_{2}^{*}=\left\{w \in \Sigma^{*} \mid \operatorname{nf}(w)=\varepsilon\right\}$.

Lemma 2 The following conditions hold of all $u, v, w, v^{\prime} \in \Sigma^{*}$ :

(i) If $v \triangleright^{*} v^{\prime} \in \bar{c} \Sigma^{*}$, then $\operatorname{nf}(v w) \in \bar{c} \Sigma^{*}$.

(ii) If $v \triangleright^{*} v^{\prime} \in \bar{d} \Sigma^{*}$, then $\operatorname{nf}(v w) \in \bar{d} \Sigma^{*}$.

(iii) If $v \triangleright^{*} v^{\prime} \in \Sigma^{*} c$, then $\operatorname{nf}(u v) \in \Sigma^{*} c$.

(iv) If $v \triangleright^{*} v^{\prime} \in \Sigma^{*} d$, then $\operatorname{nf}(u v) \in \Sigma^{*} d$.

(v) If $v \triangleright^{*} v^{\prime} \in \Sigma^{*} c \bar{d} \Sigma^{*}$, then $\operatorname{nf}(u v w) \in \Sigma^{*} c \bar{d} \Sigma^{*}$.

(vi) If $v \triangleright^{*} v^{\prime} \in \Sigma^{*} d \bar{c} \Sigma^{*}$, then $\operatorname{nf}(u v w) \in \Sigma^{*} d \bar{c} \Sigma^{*}$.

Proof (i). Since $v \triangleright^{*} v^{\prime} \in \bar{c} \Sigma^{*}$ implies $v w \triangleright^{*} v^{\prime} w \in \bar{c} \Sigma^{*}$ and, by the confluence property, $\operatorname{nf}(v w)=\operatorname{nf}\left(v^{\prime} w\right)$, it suffices to show that $z \in \bar{c} \Sigma^{*}$ implies $\operatorname{nf}(z) \in \bar{c} \Sigma^{*}$ for all $z \in \Sigma^{*}$. We prove this by induction on the number of reduction steps from $z$ to $\operatorname{nf}(z)$. Suppose $z=\bar{c} y$. If $z=\operatorname{nf}(z)$, then $\operatorname{nf}(z) \in \bar{c} \Sigma^{*}$. Otherwise, $z=\bar{c} y \triangleright^{n} \operatorname{nf}(z)$ for some $n \geq 1$. Then $\bar{c} y \triangleright x \triangleright^{n-1} \operatorname{nf}(z)=\operatorname{nf}(x)$ for some $x \in \bar{c} \Sigma^{*}$. By the induction hypothesis applied to $x$, we obtain $\operatorname{nf}(z) \in \bar{c} \Sigma^{*}$.

Part (ii)-(vi) may be proved similarly.

Lemma 3 Let $w \in \Sigma^{*}$ and suppose $\operatorname{nf}(w)=e_{1} \ldots e_{n}$ for some $e_{1}, \ldots, e_{n} \in \Sigma$. Then there exist $u_{0}, \ldots, u_{n} \in \Sigma^{*}$ such that $w=u_{0} e_{1} u_{1} \ldots e_{n} u_{n}$ and $\operatorname{nf}\left(u_{i}\right)=\varepsilon$ for $i=0, \ldots, n$. 
Proof By induction on the number of reduction steps from $w$ to $e_{1} \ldots e_{n}$.

If $K$ is a set of strings, let $\operatorname{fac}(K)$ be the set of factors of elements of $K$, i.e.,

$$
\operatorname{fac}(K)=\{v \mid u v w \in K\} .
$$

Since the relation "is a factor of" is reflexive and transitive, $\operatorname{fac}(\operatorname{fac}(K))=\operatorname{fac}(K)$ always holds.

Lemma 4 For every $w \in \operatorname{fac}\left(D_{2}^{*}\right)$, it holds that $\operatorname{nf}(w) \in(\bar{c} \mid \bar{d})^{*}(c \mid d)^{*}$.

Proof By the definition of normal form, $\mathrm{nf}(w)$ cannot contain $c \bar{c}$ or $d \bar{d}$ as a factor. Now $\operatorname{nf}(w)$ cannot contain $c \bar{d}$ or $d \bar{c}$ as a factor, either. To see this, let $u w v \in D_{2}^{*}$ and suppose $c \bar{d}$ or $d \bar{c}$ is a factor of $\operatorname{nf}(w)$. Then by Lemma 2 part (v) and (vi), $\operatorname{nf}(u w v)$ contains $c \bar{d}$ or $d \bar{c}$ as a factor, contradicting $\operatorname{nf}(u w v)=\varepsilon$. The desired conclusion now follows easily.

Lemma 5 If $v w \in D_{2}^{*}$, then $\operatorname{nf}(v) \in(c \mid d)^{*}$ and $\operatorname{nf}(w) \in(\bar{c} \mid \bar{d})^{*}$.

Proof Suppose $v w \in D_{2}^{*}$. By Lemma $4, \operatorname{nf}(v)$ and $\operatorname{nf}(w)$ both belong to $(\bar{c} \mid \bar{d})^{*}(c \mid d)^{*}$. If $\operatorname{nf}(v) \in(\bar{c} \mid \bar{d})^{+}(c \mid d)^{*}$, then by Lemma 2, part (i) and (ii), $\operatorname{nf}(v w) \in(\bar{c} \mid \bar{d}) \Sigma^{*}$, contradicting $v w \in D_{2}^{*}$. Hence $\operatorname{nf}(v) \in(c \mid d)^{*}$. Similarly, we can conclude $\operatorname{nf}(w) \in$ $(\bar{c} \mid \bar{d})^{*}$ using Lemma 2 , part (iii) and (iv).

The set $D_{2}$ of Dyck primes over $\Sigma$ is defined as $D_{2}=c D_{2}^{*} \bar{c} \mid d D_{2}^{*} \bar{d}$. It is well known and easy to see that $D_{2}^{*}$ indeed equals $\left(D_{2}\right)^{*}$.

Define context-free languages $V, L, R$ by ${ }^{6}$

$$
\begin{aligned}
& V=\varepsilon \mid L R, \\
& L=c V \bar{c}, \\
& R=d V \bar{d} .
\end{aligned}
$$

Then it is easy to see that $V \subset D_{2}^{*}, L \subset D_{2}, R \subset D_{2}$.

Lemma $6 \operatorname{fac}(V) \cap \Sigma^{2}=\{c c, c \bar{c}, \bar{c} d, d c, d \bar{d}, \bar{d} \bar{c}, \bar{d} \bar{d}\}$.

Proof First, note that $V=\varepsilon \mid L R$ implies that every $v \in V$ satisfies $v \in \varepsilon \mid c \Sigma^{*} \bar{d}$. Let $F$ be the set on the right-hand side of the equation to be proved. We can show by induction on the length of $v$ that $v \in V$ and $w \in \operatorname{fac}(v) \cap \Sigma^{2}$ imply $w \in F$. Suppose $v \in V$ and $w \in \operatorname{fac}(v) \cap \Sigma^{2}$. Then $v \in L R=c V \bar{c} d V \bar{d}$, so $v=c v_{1} \bar{c} d v_{2} \bar{d}$ for some $v_{1}, v_{2} \in$ $V$. Hence either $w \in \operatorname{fac}\left(\left\{v_{1}, v_{2}\right\}\right) \cap \Sigma^{2}$ or $w \in\{c c, c \bar{c}, \bar{d} \bar{c}, \bar{c} d, d c, d \bar{d}, \bar{d} \bar{d}\}=F$. By induction hypothesis, fac $\left(\left\{v_{1}, v_{2}\right\}\right) \cap \Sigma^{2} \subseteq F$, so it follows that $w \in F$. This establishes $\operatorname{fac}(V) \cap \Sigma^{2} \subseteq F$. To see the converse inclusion, just note that for $u=c c \bar{c} d \bar{d} \bar{c} d c \bar{c} d \bar{d} \bar{d} \in$ $V$, we have $\operatorname{fac}(u) \cap \Sigma^{2}=F$.

Lemma $7 V=\psi(H)$.

\footnotetext{
6 As usual, the sets $V, L, R$ are understood to be the components of the least solution to these equations.
} 
Proof Applying the homomorphism $\psi$ in each rule of the 3-MCFG for $H$, we get

$$
\begin{aligned}
& H\left(x_{2}\right) \leftarrow J\left(x_{1}, x_{2}, x_{3}\right) \\
& J\left(x_{1}, y_{1} c x_{2} \bar{c} d y_{2} \bar{d} x_{3}, y_{3}\right) \leftarrow J\left(x_{1}, x_{2}, x_{3}\right), J\left(y_{1}, y_{2}, y_{3}\right) \\
& J(\varepsilon, \varepsilon, \varepsilon) \leftarrow
\end{aligned}
$$

In this grammar, whenever $J\left(u_{1}, u_{2}, u_{3}\right)$ is derivable, $u_{1}=u_{3}=\varepsilon$. So the first and third arguments of $J$ can be dropped, and the grammar can be simplified to

$$
\begin{aligned}
& J(c \boldsymbol{x} \bar{c} d \boldsymbol{y} \bar{d}) \leftarrow J(\boldsymbol{x}), J(\boldsymbol{y}) \\
& J(\varepsilon) \leftarrow
\end{aligned}
$$

This is just a context-free grammar for $V$.

Lemma $8 D_{2} \cap \operatorname{fac}(V)=L \mid R$.

Proof Since $V=\varepsilon \mid L R$ and $L \mid R \subseteq D_{2}$, it is clear that $L \mid R \subseteq D_{2} \cap \operatorname{fac}(V)$.

For the converse inclusion, we prove by induction on the length of $x \in V$ that $x=u v w$ and $v \in D_{2}$ implies $v \in L \mid R$. The base case of $x=\varepsilon$ is trivial. For the induction step, let $x=c y \bar{c} d z \bar{d}$, where $y, z \in V$, and suppose $x=u v w$ and $v \in D_{2}$. We distinguish three cases.

Case 1. $v$ is a factor of $c y \bar{c}$. If $v=c y \bar{c}$, then $v \in L$, and if $v$ is a factor of $y$, then $v \in L \mid R$ by the induction hypothesis. If $v=c y^{\prime}$, where $y^{\prime}$ is a prefix of $y$, then $\operatorname{nf}(v)=\operatorname{nf}\left(c y^{\prime}\right) \in$ $c(c \mid d)^{*}$ by Lemma 5 . So $\operatorname{nf}(v) \neq \varepsilon$, contradicting $v \in D_{2}$. Likewise, if $v=y^{\prime \prime} \bar{c}$, where $y^{\prime \prime}$ is a suffix of $y$, then $\operatorname{nf}(v)=\operatorname{nf}\left(y^{\prime \prime} \bar{c}\right) \in(\bar{c} \mid \bar{d})^{*} \bar{c}$ and $\operatorname{nf}(v) \neq \varepsilon$, contradicting $v \in D_{2}$. Case 2. $v$ is a factor of $d z \bar{d}$. This case is completely analogous to Case 1 , and we can conclude $v \in L \mid R$.

Case 3. $v=v^{\prime} v^{\prime \prime}$, where $v^{\prime}$ is a non-empty suffix of $c y \bar{c}$ and $v^{\prime \prime}$ is a non-empty prefix of $d z \bar{d}$. Since $v \in D_{2}, v$ cannot equal $x=c y \bar{c} d z \bar{d}$. So either $v^{\prime}$ is a suffix of $y \bar{c}$, in which case $\operatorname{nf}(v)=\operatorname{nf}\left(v^{\prime} v^{\prime \prime}\right) \in(\bar{c} \mid \bar{d})^{*} \bar{c}(c \mid d)^{*}$ by Lemma 5, or else $v^{\prime \prime}$ is a prefix of $d z$, in which case $\operatorname{nf}(v)=\operatorname{nf}\left(v^{\prime} v^{\prime \prime}\right) \in(\bar{c} \mid \bar{d})^{*} d(c \mid d)^{*}$, again by Lemma 5. In either case, $\operatorname{nf}(v) \neq \varepsilon$, contradicting $v \in D_{2}$.

We have seen that $v \in L \mid R$ holds in all cases, and the induction step is complete.

Lemma $9 D_{2}^{*} \cap \operatorname{fac}(V)=V|L| R$.

Proof Since $V=\varepsilon \mid L R$ and $L \mid R \subseteq D_{2}$, it is clear that $V|L| R \subseteq D_{2}^{*} \cap \operatorname{fac}(V)$.

For the converse inclusion, suppose $w \in D_{2}^{*} \cap \operatorname{fac}(V)$. Since any factor of a string in $\operatorname{fac}(V)$ is itself in $\operatorname{fac}(V)$, it follows that $w \in\left(D_{2} \cap \operatorname{fac}(V)\right)^{*}$. By Lemma $8, w \in$ $(L \mid R)^{*} \cap \operatorname{fac}(V)$. Since any string in $L L|R L| R R$ has one of $\bar{c} c, \bar{d} c, \bar{d} d$ as a factor, Lemma 6 implies $(L L|R L| R R) \cap \operatorname{fac}(V)=\varnothing$. It follows that $(L \mid R)^{2} \cap \operatorname{fac}(V)=L R$ and for $n \geq 3$,

$$
\begin{aligned}
(L \mid R)^{n} \cap \operatorname{fac}(V) & =\left((L \mid R)^{2} \cap \operatorname{fac}(V)\right)(L \mid R)^{n-2} \cap \operatorname{fac}(V) \\
& =L R(L \mid R)^{n-2} \cap \operatorname{fac}(V) \\
& \subseteq L((R L \mid R R) \cap \operatorname{fac}(V))(L \mid R)^{n-3} \\
& =\varnothing .
\end{aligned}
$$


So

$$
\begin{aligned}
w & \in\left(\varepsilon|(L \mid R)|(L \mid R)^{2}\right) \cap \operatorname{fac}(V) \\
& =\varepsilon|(L \mid R)| L R \\
& =V|L| R .
\end{aligned}
$$

This proves $D_{2}^{*} \cap \operatorname{fac}(V) \subseteq V|L| R$.

Lemma 10 Let $u, w \in \Sigma^{*}$ and $v \in \Sigma^{+}$. If $u v \in V$ and $v w \in V$, then $u=w=\varepsilon$.

Proof Since $V \subset D_{2}^{*}$, Lemma 5 implies $\operatorname{nf}(v) \in(\bar{c} \mid \bar{d})^{*} \cap(c \mid d)^{*}$, and hence $\operatorname{nf}(v)=\varepsilon$. It follows that $\operatorname{nf}(u)=\operatorname{nf}(w)=\varepsilon$, too, and hence $u, v, w$ are all in $D_{2}^{*}$. By Lemma 9 $u, v, w$ are all in $V|L| R$. Since $v \neq \varepsilon$, the strings $u v$ and $v w$ are both in $V-\{\varepsilon\}=L R=$ ${ } c V \bar{c} d V \bar{d}$. So $v$ ends in $\bar{d}$ and begins in $c$. If $u \neq \varepsilon$, then $u \in L R|L| R$, so $u \in \Sigma^{*}(\bar{c} \mid \bar{d})$. This implies either $\bar{c} c$ or $\bar{d} c$ is a factor of $u v \in V$, contradicting Lemma 6 . Therefore, $u=\varepsilon$. Similarly, we can use Lemma 6 to conclude $w=\varepsilon$.

We say that a string $u$ is a proper prefix (proper suffix) of a string $v$ if $u$ is a prefix (suffix) of $v$ and $u \neq v$. Lemma 10 implies that no proper prefix or proper suffix of a string in $V$ can belong to $V$, which is to say that $V$ is both prefix-free and suffix-free.

\section{Lemma 11}

$$
\begin{aligned}
\operatorname{fac}(V) \subseteq & (V|L| R) \mid \\
& (V \mid R)(\bar{c} R \mid \bar{d})^{*}(\bar{c}|\bar{c} R| \bar{d}) \mid \\
& (c|L d| d)(c \mid L d)^{*}(V \mid L) \mid \\
& (V \mid R)(\bar{c} R \mid \bar{d})^{*} \bar{c} d(c \mid L d)^{*}(V \mid L) .
\end{aligned}
$$

Proof Suppose $w \in \operatorname{fac}(V)$. By Lemma $4 \operatorname{nf}(w) \in(\bar{c} \mid \bar{d})^{m}(c \mid d)^{n}$ for some $m, n \geq 0$, and by Lemma 3, there are strings $u_{0}, \ldots, u_{m+n}$ such that $\operatorname{nf}\left(u_{i}\right)=\varepsilon$ for each $i=$ $0, \ldots, m+n$ and

$$
w \in u_{0}(\bar{c} \mid \bar{d}) u_{1} \ldots(\bar{c} \mid \bar{d}) u_{m}(c \mid d) u_{m+1} \ldots(c \mid d) u_{m+n} .
$$

Since $u_{i}$ is a factor of $w \in \operatorname{fac}(V), u_{i} \in D_{2}^{*} \cap \operatorname{fac}(V)$. Lemma 9 then implies $u_{i} \in V \mid$ $L \mid R$.

By Lemma 6 , each of the following sets is disjoint from fac $(V)$ :

$$
\begin{array}{ll}
\bar{c}(\bar{c} \mid \bar{d}), & (c \mid d) d, \\
\bar{d}(c \mid d), & (\bar{c} \mid \bar{d}) c .
\end{array}
$$

This implies that the following conditions hold:

$$
\begin{gathered}
u_{0} \in V \mid R \text { if } m \geq 1, \\
u_{m+n} \in V \mid L \text { if } n \geq 1,
\end{gathered}
$$

$u_{i} \in \varepsilon \mid R$ if $u_{i}$ is preceded by $\bar{c}$,

$u_{i} \in R$ if $u_{i}$ is preceded by $\bar{c}$ and is followed by $\bar{c}$ or $\bar{d}$,

$$
u_{i}=\varepsilon \text { if } u_{i} \text { is preceded by } \bar{d},
$$

$u_{i}=\varepsilon$ if $u_{i}$ is followed by $c$,

$$
u_{i} \in \varepsilon \mid L \text { if } u_{i} \text { is followed by } d,
$$

$u_{i} \in L$ if $u_{i}$ is preceded by $c$ or $d$ and is followed by $d$. 
Case 1. $m=n=0$. Then $w=u_{0} \in V|L| R$.

Case 2. $m \geq 1, n=0$. Then $w \in u_{0}(\bar{c} \mid \bar{d}) u_{1} \ldots(\bar{c} \mid \bar{d}) u_{m}$. By (2), (4), (5), and (6), we get $w \in(V \mid R)(\bar{c} R \mid \bar{d})^{*}(\bar{c}|\bar{c} R| \bar{d})$.

Case 3. $m=0, n \geq 1$. Then $w \in u_{0}(c \mid d) \ldots u_{n-1}(c \mid d) u_{n}$. By (3), (7), (8), and (9), we get $w \in(c|L d| d)(c \mid L d)^{*}(V \mid L)$.

Case 4. $m, n \geq 1$. By (4), (6), 7), and (8), we see that $u_{m}=\varepsilon$. Since $(\bar{c} c|\bar{d} c| \bar{d} d) \cap$ $\operatorname{fac}(V)=\varnothing$,

$$
w \in u_{0}(\bar{c} \mid \bar{d}) u_{1} \ldots(\bar{d} \mid \bar{d}) u_{m-1} \bar{c} d u_{m+1}(c \mid d) \ldots u_{m+n-1}(c \mid d) u_{m+n} .
$$

By [2], 3, , 5), 6, (7), and 99, we see that $w \in(V \mid R)(\bar{c} R \mid \bar{d})^{*} \bar{c} d(c \mid L d)^{*}(V \mid L)$.

This proves the lemma.

Lemma 12 If $w \in \Sigma^{+}$and $w w \in \operatorname{fac}(V)$, then one of the following conditions holds:

(i) $w \in(\bar{c} R \mid \bar{d})^{+}$.

(ii) $w \in R(\bar{c} R \mid \bar{d})^{*} \bar{c}$.

(iii) $w \in(c \mid L d)^{+}$.

(iv) $w \in d(c \mid L d)^{*} L$.

(v) $w \in(V \mid R)(\bar{c} R \mid \bar{d})^{m} \bar{c} d(c \mid L d)^{n}(V \mid L)$ for some $m, n \geq 0$ such that $m \neq n$.

Proof Suppose $w \neq \varepsilon$ and $w w \in \operatorname{fac}(V)$. Since $w \in$ fac $(V)$, by Lemma 11 ,

$$
\begin{aligned}
\operatorname{fac}(V) \subseteq & (V|L| R) \mid \\
& (V \mid R)(\bar{c} R \mid \bar{d})^{*}(\bar{c}|\bar{c} R| \bar{d}) \mid \\
& (c|L d| d)(c \mid L d)^{*}(V \mid L) \mid \\
& (V \mid R)(\bar{c} R \mid \bar{d})^{*} \bar{c} d(c \mid L d)^{*}(V \mid L) .
\end{aligned}
$$

Case 1. $w \in V|L| R$. Since $w \neq \varepsilon, w \in L R|L| R$. It follows that $w w$ has one of $\bar{d} c, \bar{c} c, \bar{d} d$ as a factor, which contradicts $w w \in$ fac $(V)$ by Lemma 6 . So this case is impossible.

Case 2. $w \in(V \mid R)(\bar{c} R \mid \bar{d})^{*}(\bar{c}|\bar{c} R| \bar{d})$. If $w$ starts in $c$, then $w w$ contains either $\bar{c} c$ or $\bar{d} c$ as a factor, which contradicts $w w \in$ fac $(V)$ by Lemma 6 . So

$$
w \in(\varepsilon \mid R)(\bar{c} R \mid \bar{d})^{*}(\bar{c}|\bar{c} R| \bar{d}) .
$$

Case 2.1. $w \in(\bar{c} R \mid \bar{d})^{*}(\bar{c}|\bar{c} R| \bar{d})$. If $w$ ends in $\bar{c}, w w$ contains either $\bar{c} \bar{c}$ or $\bar{c} \bar{d}$ as a factor, which contradicts $w w \in \operatorname{fac}(V)$ by Lemma 6 . So in this case $w \in(\bar{c} R \mid \bar{d})^{*}(\bar{c} R \mid$ $\bar{d})=(\bar{c} R \mid \bar{d})^{+}$.

Case 2.2. $w \in R(\bar{c} R \mid \bar{d})^{*}(\bar{c}|\bar{c} R| \bar{d})$ In this case, $w$ starts in $d$. If $w$ ends in $\bar{d}$, then $w w$ contains either $\bar{d} d$ as a factor, contradicting $w w \in \operatorname{fac}(V)$ by Lemma 6 So in this case $w \in R(\bar{c} R \mid \bar{d})^{*} \bar{c}$.

Case 3. $w \in(c|L d| d)(c \mid L d)^{*}(V \mid L)$. This case is exactly symmetric to Case 2, and we can conclude $w \in(c \mid L d)^{+}$or $w \in d(c \mid L d)^{*} L$. 
Case 4. $w \in(V \mid R)(\bar{c} R \mid \bar{d})^{*} \bar{c} d(c \mid L d)^{*}(V \mid L)$. Let $m, n \geq 0$ be such that

$$
w \in(V \mid R)(\bar{c} R \mid \bar{d})^{m} \bar{c} d(c \mid L d)^{n}(V \mid L) .
$$

We show that $m \neq n$. Suppose, by way of contradiction, $m=n$. Then $w w$ contains a factor $u$ that belongs to

$$
d(c \mid L d)^{n}(V \mid L)(V \mid R)(\bar{c} R \mid \bar{d})^{n} \bar{c} .
$$

Note that

$$
u \triangleright^{*} u^{\prime} \in d(c \mid d)^{n}(\bar{c} \mid \bar{d})^{n} \bar{c} .
$$

It is easy to see from this that $\operatorname{nf}(u)$ has either $c \bar{d}$ or $d \bar{c}$ as a factor. But since $u$ is a factor of $w w, u \in \operatorname{fac}(V) \subseteq \operatorname{fac}\left(D_{2}^{*}\right)$. By Lemma $4 \operatorname{nf}(u) \in(\bar{c} \mid \bar{d})^{*}(c \mid d)^{*}$, a contradiction.

We have proved that one of (i)-(v) holds in each case.

\subsection{Properties of the 3-MCFL $H$}

Lemma 12 immediately yields a necessary condition for membership in $\left\{w \in \hat{\Sigma}^{+}\right.$ $w w \in \operatorname{fac}(H)\}$. For $w$ to be in this set, it must be that $\psi(w) \psi(w)=\psi(w w) \in$ $\psi(\operatorname{fac}(H))=\operatorname{fac}(\psi(H))=\operatorname{fac}(V)$, so either $\psi(w)=\varepsilon$, in which case $w \in a^{+} \mid b^{+}$, or $\psi(w)$ must satisfy one of the five conditions in Lemma 12. This will be used in the next section to give a necessary condition for membership in

$$
\left\{w \in \hat{\Sigma}^{+} \mid w w \in \operatorname{fac}(H)\right\} \cap \operatorname{fac}\left(\left\{v_{n} \mid n \in \mathbb{N}\right\}\right),
$$

where $\left\{v_{n} \mid n \in \mathbb{N}\right\}$ is a certain infinite subset of $H$. In this section, we establish some general properties of $H$ that will be useful in the next section.

Lemma 13 For every $v \in V$, there is a unique string $w \in H$ such that $\psi(w)=v$.

Proof We prove by induction on the length of $v \in V$ that there is a unique triple $\left(w_{1}, w_{2}, w_{3}\right)$ such that $J\left(w_{1}, w_{2}, w_{3}\right)$ is derivable and $\psi\left(w_{2}\right)=v$. It is clear from the grammar for $H$ that $\vdash J\left(w_{1}, w_{2}, w_{3}\right)$ and $\psi\left(w_{2}\right)=\varepsilon$ imply $w_{1}=a, w_{2}=\varepsilon, w_{3}=b$. This takes care of the case $v=\varepsilon$. Now suppose $v \in L R$. Then $v=c u_{1} \bar{c} d u_{2} \bar{d}$ for some $u_{1}, u_{2} \in V$. Note that the choice of $u_{1}$ and $u_{2}$ is unique. For, if $v=c u_{1}^{\prime} \bar{c} d u_{2}^{\prime} \bar{d}$ for some $u_{1}^{\prime}, u_{2}^{\prime} \in V$, then $u_{1}^{\prime}$ either is a prefix of $u_{1}$ or contains $u_{1}$ as a prefix, which implies $u_{1}=u_{1}^{\prime}$ by Lemma 10 Similarly, $u_{2}^{\prime}$ either is a suffix of $u_{2}$ or contains $u_{2}$ as a suffix, and it follows that $u_{2}=u_{2}^{\prime}$. If $\vdash J\left(w_{1}, w_{2}, w_{3}\right)$ and $\psi\left(w_{2}\right)=v$, then $w_{2}$ cannot be $\varepsilon$ and there must be some $x_{1}, y_{1} \in a^{+}, x_{2}, y_{2} \in H$, and $x_{3}, y_{3} \in b^{+}$such that

$$
\begin{gathered}
\vdash J\left(x_{1}, x_{2}, x_{3}\right), \\
\vdash J\left(y_{1}, y_{2}, y_{3}\right), \\
w_{1}=a x_{1}, \\
w_{2}=y_{1} c x_{2} \bar{c} d y_{2} \bar{d} x_{3}, \\
w_{3}=y_{3} b .
\end{gathered}
$$


Since $\psi\left(w_{2}\right)=v$, we have $c \psi\left(x_{2}\right) \bar{c} d \psi\left(y_{2}\right) \bar{d}=c u_{1} \bar{c} d u_{2} \bar{d}$. Since $x_{2}, y_{2} \in H$, both $\psi\left(x_{2}\right)$ and $\psi\left(y_{2}\right)$ are in $\psi(H)=V$. It follows that $\psi\left(x_{2}\right)=u_{1}$ and $\psi\left(y_{2}\right)=u_{2}$. By induction hypothesis, $\left(x_{1}, x_{2}, x_{3}\right)$ and $\left(y_{1}, y_{2}, y_{3}\right)$ are uniquely determined by $u_{1}$ and $u_{2}$, respectively. Since $u_{1}$ and $u_{2}$ are uniquely determined by $v$, the triple $\left(w_{1}, w_{2}, w_{3}\right)$ is uniquely determined by $v$.

Let $\$$ be a symbol not in $\hat{\Sigma}$. We use this symbol to mark the beginning and end of a string in $H$.

Lemma $14 \operatorname{fac}(\$ H \$) \cap(\{\$\} \cup \hat{\Sigma})^{2}=\{\$ \$, \$ a, a a, a c, b \$, b b, b \bar{c}, b \bar{d}, c a, c \bar{c}, \bar{c} d, d a, d \bar{d}, \bar{d} b\}$.

Proof Let $F$ denote the set on the right-hand side of the equation. We prove by induction on the length of $u_{2}$ that $\vdash J\left(u_{1}, u_{2}, u_{3}\right)$ implies fac $\left(\$ u_{2} \$\right) \cap(\{\$\} \cup \hat{\Sigma})^{2} \subseteq F$. For the induction basis, observe that $\operatorname{fac}(\$ \varepsilon \$) \cap(\{\$\} \cup \hat{\Sigma})^{2}=\{\$ \$\} \subseteq F$. Now suppose for some $x_{1}, x_{2}, x_{3}, y_{1}, y_{2}, y_{3}$ such that $\vdash J\left(x_{1}, x_{2}, x_{3}\right)$ and $\vdash J\left(y_{1}, y_{2}, y_{3}\right)$, we have $u_{1}=a x_{1}, u_{2}=y_{1} c x_{2} \bar{c} d y_{2} \bar{d} x_{3}, u_{3}=y_{3} b$. It follows from the induction hypothesis applied to $x_{2}$ and $y_{2}$ that

$$
\begin{aligned}
\operatorname{fac}\left(c x_{2} \bar{c}\right) \cap \hat{\Sigma}^{2} & \subseteq(F-\{\$ \$, \$ a, b \$\}) \cup\{c \bar{c}, c a, b \bar{c}\} \\
& =F-\{\$ \$, \$ a, b \$\} \\
\operatorname{fac}\left(d y_{2} \bar{d}\right) \cap \hat{\Sigma}^{2} & \subseteq(F-\{\$ \$, \$ a, b \$\}) \cup\{d \bar{d}, d a, b \bar{d}\} \\
& =F-\{\$ \$, \$ a, b \$\} .
\end{aligned}
$$

Since $y_{1} \in a^{+}$and $x_{3} \in b^{+}$, we get

$$
\begin{aligned}
& \operatorname{fac}\left(\$ y_{1} c x_{2} \bar{c} d y_{2} \bar{d} x_{3} \$\right) \cap(\{\$\} \cup \hat{\Sigma})^{2} \\
& \subseteq\{\$ a, a a, a c\} \cup\left(\operatorname{fac}\left(c x_{2} \bar{c}\right) \cap \hat{\Sigma}^{2}\right) \cup\{\bar{c} d\} \cup\left(\operatorname{fac}\left(d y_{2} \bar{d}\right) \cap \hat{\Sigma}^{2}\right) \cup\{\bar{d} b, b b, b \$\} \\
& \subseteq F
\end{aligned}
$$

Therefore, fac $(\$ H \$) \cap(\{\$\} \cup \hat{\Sigma})^{2} \subseteq F$. To see the converse inclusion, note that for $v=a a c a c \bar{c} d \bar{d} b \bar{c} d a c \bar{c} d \bar{d} b \bar{d} b b \in H$, we have fac $(\$ \nu \$) \cap(\{\$\} \cup \hat{\Sigma})^{2}=F-\{\$ \$\}$.

Lemma 15 Let $u, w \in \hat{\Sigma}^{*}$ and $v \in \hat{\Sigma}^{+}$. If $u v \in H$ and $v w \in H$, then $u=w=\varepsilon$.

Proof Since $v \neq \varepsilon$, Lemma 14 implies that both $u v$ and $v w$ start in $a$ and end in $b$. Hence $v$ starts in $a$ and ends in $b$. By Lemma 14, the only symbols that can follow $a$ in $v$ are $a$ and $c$, and the only symbols that can precede $b$ in $v$ are $b$ and $\bar{d}$. So $v \in$ $a^{+} c \hat{\Sigma}^{*} \bar{d} b^{+}$. Since $\psi(v) \neq \varepsilon$ and $\psi(u v)$ and $\psi(v w)$ are both in $\psi(H)=V$, Lemma 10 implies that $\psi(u)=\psi(w)=\varepsilon$. Hence $\psi(u v)=\psi(v w)$, and by Lemma $13, u v=v w$. But $\psi(u)=\psi(w)=\varepsilon$ implies $u \in a^{*}$ and $w \in b^{*}$, and it easily follows that $u=w=\varepsilon$.

Lemma 15 implies that $H$ is both prefix-free and suffix-free.

Lemma 16 (i) $H \subseteq \varepsilon \mid\left(a^{+} c\right)^{+} \bar{c} \hat{\Sigma}^{*} d\left(\bar{d} b^{+}\right)^{+}$.

(ii) If $\vdash J\left(u_{1}, u_{2}, u_{3}\right)$ and $u_{2} \in\left(a^{*} c\right)^{k}\left(\bar{c} \hat{\Sigma}^{*} d \mid \varepsilon\right)\left(\bar{d} b^{*}\right)^{l}$, then $u_{1}=a^{k+1}$ and $u_{3}=b^{l+1}$. 
Proof (i). Suppose $v \neq \varepsilon$ and $v \in H$. We reason using Lemma 14 . The first symbol of $v$ must be $a$. Also, in $v$, the only symbols that can follow $a$ are $a$ and $c$, and the only symbols that can follow $c$ are $a$ and $\bar{c}$. Since the last symbol of $v$ must be $b$, it follows that $v$ has a prefix that belongs to $\left(a^{+} c\right)^{+} \bar{c}$. By a symmetric reasoning, $v$ has a suffix that belongs to $d\left(\bar{d} b^{+}\right)^{+}$. Therefore, $v \in\left(a^{+} c\right)^{+} \bar{c} \hat{\Sigma}^{*} d\left(\bar{d} b^{+}\right)^{+}$.

(ii). We prove this par 7 by induction on the length of $u_{2}$. Suppose $\vdash J\left(u_{1}, u_{2}, u_{3}\right)$. If $u_{2}=\varepsilon \in\left(a^{*} c\right)^{0}\left(\bar{c} \hat{\Sigma}^{*} d \mid \varepsilon\right)\left(\bar{d} b^{*}\right)^{0}$, then we must have $u_{1}=a^{1}$ and $u_{3}=b^{1}$. If $u \neq \varepsilon$, then there exist $x_{1}, x_{2}, x_{3}, y_{1}, y_{2}, y_{3}$ such that $\vdash J\left(x_{1}, x_{2}, x_{3}\right), \vdash J\left(y_{1}, y_{2}, y_{3}\right), u_{1}=a x_{1}$, $u_{2}=y_{1} c x_{2} \bar{c} d y_{2} \bar{d} x_{3}, u_{3}=y_{3} b$. Suppose $u_{2} \in\left(a^{*} c\right)^{k}\left(\bar{c} \hat{\Sigma}^{*} d \mid \varepsilon\right)\left(\bar{d} b^{*}\right)^{l}$. Since $y_{1} \in a^{*}$ and $x_{3} \in b^{*}$, we have $k, l \geq 1$, and part (i) of the lemma implies that for some $m, n \geq 0$,

$$
\begin{aligned}
& x_{2} \in\left(a^{*} c\right)^{k-1}\left(\bar{c} \hat{\Sigma}^{*} d \mid \varepsilon\right)\left(\bar{d} b^{*}\right)^{m}, \\
& y_{2} \in\left(a^{*} c\right)^{n}\left(\bar{c} \hat{\Sigma}^{*} d \mid \varepsilon\right)\left(\bar{d} b^{*}\right)^{l-1} .
\end{aligned}
$$

By induction hypothesis, $x_{1}=a^{k}$ and $y_{3}=b^{l}$. Therefore, $u_{1}=a^{k+1}$ and $u_{3}=b^{l+1}$.

Note that by Lemma 14 , in any string in $H, \bar{c}$ always precedes $d$ and $d$ always follows $\bar{c}$.

Lemma 17 For all $u, v \in \hat{\Sigma}^{*}$, the following conditions hold:

(i) If $u c v \in H$, then for some $k \geq 1$,

$$
u \in\left(\varepsilon \mid \hat{\Sigma}^{*}(c \mid d)\right) a^{k}, \quad a^{k} c v \in H\left(\varepsilon \mid(\bar{c} \mid \bar{d}) \hat{\Sigma}^{*}\right)
$$

(ii) If $u \bar{d} v \in H$, then for some $l \geq 1$,

$$
u \bar{d} b^{l} \in\left(\varepsilon \mid \hat{\Sigma}^{*}(c \mid d)\right) H, \quad v \in b^{l}\left(\varepsilon \mid(\bar{c} \mid \bar{d}) \hat{\Sigma}^{*}\right) .
$$

(iii) If $u \bar{c} d v \in H$, then for some $k, l \geq 1$,

$$
u \in\left(\varepsilon \mid \hat{\Sigma}^{*}(c \mid d)\right) a^{k} c H, \quad v \in H \bar{d} b^{l}\left(\varepsilon \mid(\bar{c} \mid \bar{d}) \hat{\Sigma}^{*}\right) .
$$

Proof Each of the three conditions can be proved by easy induction on the combined length of $u$ and $v$. We only prove (i). Suppose $u c v \in H$. Since $u c v \neq \varepsilon$, there must be $y_{1} \in a^{+}, x_{2}, y_{2} \in H$, and $x_{3} \in b^{+}$such that $u c v=y_{1} c x_{2} \bar{c} d y_{2} \bar{d} x_{3}$. If $u=y_{1}$, then we can take $a^{k}=y_{1}$. Otherwise, either $u=y_{1} c x_{2}^{\prime}, v=x_{2}^{\prime \prime} \bar{c} d y_{2} \bar{d} x_{3}$ for some $x_{2}^{\prime}, x_{2}^{\prime \prime}$ such that $x_{2}=$ $x_{2}^{\prime} c x_{2}^{\prime \prime}$, or $u=y_{1} c x_{2} \bar{c} d y_{2}^{\prime}, v=y_{2}^{\prime \prime} \bar{d} x_{3}$ for some $y_{2}^{\prime}, y_{2}^{\prime \prime}$ such that $y_{2}=y_{2}^{\prime} c y_{2}^{\prime \prime}$. In the former case, we can apply the induction hypothesis to $x_{2}^{\prime}, x_{2}^{\prime \prime}$ and obtain $x_{2}^{\prime} \in\left(\varepsilon \mid \hat{\Sigma}^{*}(c \mid d)\right) a^{k}$ and $a^{k} c x_{2}^{\prime \prime} \in H\left(\varepsilon \mid(\bar{c} \mid \bar{d}) \hat{\Sigma}^{*}\right)$ for some $k \geq 1$. It follows that $u=y_{1} c x_{2}^{\prime} \in \hat{\Sigma}^{*}(c \mid d) a^{k}$ and $a^{k} c v=a^{k} c x_{2}^{\prime \prime} \bar{c} d y_{2} \bar{d} x_{3} \in H(\bar{c} \mid \bar{d}) \hat{\Sigma}^{*}$. In the latter case, we can apply the induction hypothesis to $y_{2}^{\prime}, y_{2}^{\prime \prime}$ and obtain $y_{2}^{\prime} \in\left(\varepsilon \mid \hat{\Sigma}^{*}(c \mid d)\right) a^{k}$ and $a^{k} c y_{2}^{\prime \prime} \in H\left(\varepsilon \mid(\bar{c} \mid \bar{d}) \hat{\Sigma}^{*}\right)$ for some $k \geq 1$, and we can similarly infer $u=y_{1} c x_{2} \bar{c} d y_{2}^{\prime} \in \hat{\Sigma}^{*}(c \mid d) a^{k}$ and $a^{k} c v=$ $a^{k} c y_{2}^{\prime \prime} \bar{d} x_{3} \in H(\bar{c} \mid \bar{d}) \hat{\Sigma}^{*}$.

Lemma 18 Suppose $w \in \operatorname{fac}(\$ H \$)$. For all $k, l \geq 0$, the following conditions hold:

\footnotetext{
7 By part (i), part (ii) can be equivalently stated with $a^{+}$and $b^{+}$in place of $a^{*}$ and $b^{*}$, but it will turn out to be slightly more convenient in this form.
} 
(i) $w \in(\$|c| d) a^{k} c H \bar{c} d\left(a^{*} c\right)^{l}(\bar{c} \mid \bar{d})$ implies $k=l+1$.

(ii) $w \in(c \mid d)\left(\bar{d} b^{*}\right)^{k} \bar{c} d H \bar{d} b^{l}(\bar{c}|\bar{d}| \$)$ implies $k+1=l$.

Proof We only prove part (i), since part (ii) is exactly symmetric. Suppose that $w \in$ $\operatorname{fac}(\$ H \$)$ and for some $u \in H$,

$$
\begin{aligned}
w & \in(\$|c| d) w^{\prime}, \\
w^{\prime} & \in a^{k} c u \bar{c} d\left(a^{*} c\right)^{l}(\bar{c} \mid \bar{d}) .
\end{aligned}
$$

By Lemma 17, part (i), there is a string $z \in H$ such that $w^{\prime}$ is a prefix of some string in $z\left(\varepsilon \mid(\bar{c} \mid \bar{d}) \hat{\Sigma}^{*}\right)$. Since $w^{\prime}$ starts in $a$ or $c$, the string $z$ cannot be $\varepsilon$. Hence there are some strings $x_{1}, x_{2}, x_{3}, y_{1}, y_{2}, y_{3}$ such that $\vdash J\left(x_{1}, x_{2}, x_{3}\right), \vdash J\left(y_{1}, y_{2}, y_{3}\right)$, and $z=$ $y_{1} c x_{2} \bar{c} d y_{2} \bar{d} x_{3}$. So

$$
w^{\prime} \text { is a prefix of some string in } y_{1} c x_{2} \bar{c} d y_{2} \bar{d} x_{3}\left(\varepsilon \mid(\bar{c} \mid \bar{d}) \hat{\Sigma}^{*}\right) .
$$

Note that $x_{1}, y_{1} \in a^{+}$and $x_{3}, y_{3} \in b^{+}$. So clearly, $y_{1}=a^{k}$, and either $x_{2} \bar{c}$ is a prefix of $u \bar{c}$, or else $u \bar{c}$ is a prefix of $x_{2} \bar{c}$. Since $u \in H$ and $x_{2} \in H$, neither $u$ nor $x_{2}$ can start in $\bar{c}$. It follows that $u=\varepsilon$ if and only if $x_{2}=\varepsilon$. If $u \neq \varepsilon$ and $x_{2} \neq \varepsilon$, then either $u$ is a non-empty prefix of $x_{2}$ or vice versa, and Lemma 15 implies that $u=x_{2}$. Hence we always have $a^{k} c u \bar{c} d=y_{1} c x_{2} \bar{c} d$. It follows that $y_{2} \bar{d}$ has a prefix belonging to $\left(a^{*} c\right)^{l}(\bar{c} \mid \bar{d})$. Since $y_{2} \in H$, by Lemma 16 , part (i), either $l=0$ and $y_{2}=\varepsilon$ or $l \geq 1$ and $y_{2}$ has a prefix belonging to $\left(a^{*} c\right)^{l} \bar{c}$. We can now apply Lemma 16 , part (ii), to $J\left(y_{1}, y_{2}, y_{3}\right)$ and obtain $k=l+1$.

\subsection{Almost Anti-iterative Elements of $H$}

Given a language $K$ and a string $w \in K$, an iteration tuple for $w$ in $K$ is a tuple of strings $\left(u_{0}, w_{1}, u_{1}, \ldots, w_{k}, u_{k}\right)$ such that

- $w=u_{0} w_{1} u_{1} \ldots w_{k} u_{k}$

$-w_{1} \ldots w_{k} \neq \varepsilon$, and

- $u_{0} w_{1}^{i} u_{1} \ldots w_{k}^{i} u_{k} \in K$ for all $i \geq 0$.

The notion of an iteration tuple is a generalization of the notion of an iterative pair [1]. A language $K$ is said to be $k$-iterative if all but finitely many strings in $K$ have an iteration tuple $\left(u_{0}, w_{1}, u_{1}, \ldots, w_{k}, u_{k}\right)$ (of length $\left.2 k+1\right)$ in $K$. We simply say that $K$ is iterative if all but finitely many strings in $K$ have an iteration tuple (of any length) in $K$. (Iterativity is a slight weakening of the property Groenink [5],4] called finite pumpability.)

We prove a theorem that implies that the language $H$ is not iterative. In fact, the theorem states something much stronger. We say that a string $v \in K$ is anti-iterative in $K$ if $v=u_{0} w_{1} u_{1} \ldots w_{k} u_{k}$ and $w_{1} \ldots w_{k} \neq \varepsilon$ (for any $k \geq 1$ ) imply $u_{0} w_{1}^{i} u_{1} \ldots w_{k}^{i} u_{k} \notin K$ for all $i>1$. We say that $v \in K$ is almost anti-iterative in $K$ if $v=u_{0} w_{1} u_{1} \ldots w_{k} u_{k}$ and $w_{1} \ldots w_{k} \neq \varepsilon$ (for any $k \geq 1$ ) imply that there is at most one natural number $i>1$ such that $u_{0} w_{1}^{i} u_{1} \ldots w_{k}^{i} u_{k} \in K$. Clearly, if $v$ is almost anti-iterative in $K$, then there is no iteration tuple for $v$ in $K$. 
Now for each $n \geq 0$, define a string $v_{n} \in H$ as follows:

$$
\begin{aligned}
v_{0} & =\varepsilon, \\
v_{n+1} & =a^{n+1} c v_{n} \bar{c} d v_{n} \bar{d} b^{n+1} .
\end{aligned}
$$

It is easy to see $\vdash J\left(a^{n+1}, v_{n}, b^{n+1}\right)$ for all $n \in \mathbb{N}$. The strings $v_{n}$ are precisely those elements of $H$ that have a derivation tree whose immediate subtree is a perfect binary tree. We will show that each $v_{n}$ is almost anti-iterative in $H$.

We start with some lemmas (Lemmas 19,22) stating some general properties of the strings $v_{n}$ that are intuitively obvious from the way they are defined. We give a fairly rigorous proof to each of these lemmas.

Lemma $19 v_{n} \in\left(a^{+} c\right)^{n}\left(\bar{c} \hat{\Sigma}^{*} d \mid \varepsilon\right)\left(\bar{d} b^{+}\right)^{n}$ for all $n$.

Proof For $n=0, v_{0}=\varepsilon=\left(a^{+} c\right)^{0} \varepsilon\left(\bar{d} b^{+}\right)^{0}$, so the desired condition holds. For $n \geq 1$, we prove by induction on $n$ that $v_{n} \in\left(a^{+} c\right)^{n} \bar{c}^{*} \hat{\Sigma}^{*} d\left(\bar{d} b^{+}\right)^{n}$. For $n=1, v_{1}=a c \bar{c} d \bar{d} b \in$ $\left(a^{+} c\right)^{1} \bar{c} \hat{\Sigma}^{*} d\left(\bar{d} b^{+}\right)^{1}$. For $n \geq 2$, assume $v_{n-1} \in\left(a^{+} c\right)^{n-1} \bar{c} \hat{\Sigma}^{*} d\left(\bar{d} b^{+}\right)^{n-1}$. Then $v_{n}=$ $a^{n} c v_{n-1} \bar{c} d v_{n-1} \bar{d} b^{n} \in\left(a^{+} c\right)^{n} \bar{c} \hat{\Sigma}^{*} d\left(\bar{d} b^{+}\right)^{n}$.

Lemma $20 \operatorname{fac}\left(\left\{v_{n} \mid n \in \mathbb{N}\right\}\right) \cap H=\left\{v_{n} \mid n \in \mathbb{N}\right\}$.

Proof Clearly, it suffices to show the inclusion, $\operatorname{fac}\left(\left\{v_{n} \mid n \in \mathbb{N}\right\}\right) \cap H \subseteq\left\{v_{n} \mid n \in \mathbb{N}\right\}$. We prove by induction on $n \in \mathbb{N}$ that $w \in$ fac $\left(v_{n}\right) \cap H$ implies $w=v_{k}$ for some $k \leq n$. Since $v_{0}=\varepsilon \in H$, the induction basis is immediate. Now assume $w \in H$ and $w$ is a factor of $v_{n+1}=a^{n+1} c v_{n} \bar{c} d v_{n} \bar{d} b^{n+1}$. By Lemma 16 part (i), either $w=\varepsilon$ or $w \in$ $\left(a^{+} c\right)^{+} \bar{c} \hat{\Sigma}^{*} d\left(\bar{d} b^{+}\right)^{+}$. If $w=\varepsilon$, then $w=v_{0}$. It remains to consider the case where $w \in$ $\left(a^{+} c\right)^{+} \bar{c} \hat{\Sigma}^{*} d\left(\bar{d} b^{+}\right)^{+}$. If $\psi(w)=\psi\left(v_{n+1}\right)$, then $w=v_{n+1}$ by Lemma 13. If $\psi(w) \neq$ $\psi\left(v_{n+1}\right)$, then either $w$ is a factor of $v_{n} \bar{c} d v_{n} \bar{d} b^{n+1}$ or $w$ is a factor of $a^{n+1} c v_{n} \bar{c} d v_{n}$.

Case 1. $w$ is a factor of $v_{n} \bar{c} d v_{n} \bar{d} b^{n+1}$. Since $w$ starts in $a$, there must be a non-empty suffix $y$ of $v_{n}$ starting in $a$ such that $w$ is a prefix of $y \bar{c} d v_{n} \bar{d} b^{n+1}$ or of $y \bar{d} b^{n+1}$. Since $y$ is a suffix of $v_{n} \in H$, Lemma 15 implies that $y$ cannot be a proper prefix of any element of $H$. Since $w \in H$, it follows that $y$ is not a proper prefix of $w$. Since $w$ is a prefix of $y \bar{c} d v_{n} \bar{d} b^{n+1}$ or of $y \bar{d} b^{n+1}, w$ must be a prefix of $y$.

Case 2. $w$ is a factor of $a^{n+1} c v_{n} \bar{c} d v_{n}$. Since $w$ ends in $b$, there must be a non-empty prefix $x$ of $v_{n}$ ending in $b$ such that $w$ is a suffix of $a^{n+1} c x$ or of $a^{n+1} c v_{n} \bar{c} d x$. By an analogous reasoning to the previous case, we can conclude that $w$ is a suffix of $x$.

In both cases, $w$ is a factor of $v_{n}$, and the induction hypothesis gives $w=v_{k}$ for some $k \leq n$.

Lemma 21 Suppose $w \in \operatorname{fac}\left(\$\left\{v_{n} \mid n \in \mathbb{N}\right\} \$\right)$. For all $k, l \geq 0$, the following conditions hold:

(i) $w \in(\$|c| d) a^{k}(c \mid c H \bar{c} d) a^{l}(c|\bar{c}| \bar{d})$ implies $k=l+1$.

(ii) $w \in(c|d| \bar{d}) b^{k}(\bar{c} d H \bar{d} \mid \bar{d}) b^{l}(\bar{c}|\bar{d}| \$)$ implies $k+1=l$.

(iii) $w \in(c \mid \bar{d}) b^{k} \bar{c} d a^{l}(c \mid \bar{d})$ implies $k=l$. 
Proof (i). Suppose $u w v=\$ v_{n} \$$ and

$$
\begin{aligned}
w & \in(\$|c| d) w^{\prime}, \\
w^{\prime} & \in a^{k}(c \mid c H \bar{c} d) a^{l}(c|\bar{c}| \bar{d}) .
\end{aligned}
$$

By Lemma 17 part (i), $k \geq 1$ and there is a $z \in H$ such that $w^{\prime} v \in z\left(\varepsilon \mid(\bar{c} \mid \bar{d}) \hat{\Sigma}^{*}\right) \$$. Since $w^{\prime}$ starts in $a, z \neq \varepsilon$. Lemma 20 implies that $z=v_{k}=a^{k} c v_{k-1} \bar{c} d v_{k-1} \bar{d} b^{k}$. So

$$
w^{\prime} v \in a^{k} c v_{k-1} \bar{c} d v_{k-1} \bar{d} b^{k}\left(\varepsilon \mid(\bar{c} \mid \bar{d}) \hat{\Sigma}^{*}\right) \$ .
$$

By (10), either $w^{\prime} \in a^{k} c a^{l}(c|\bar{c}| \bar{d})$ or $w^{\prime} \in a^{k} c H \bar{c} d a^{l}(c|\bar{c}| \bar{d})$.

Case 1. $w^{\prime} \in a^{k} c a^{l}(c|\bar{c}| \bar{d})$. Then either $k=1, v_{k-1}=\varepsilon, l=0$, and $w^{\prime}=a^{k} c \bar{c}$, or $k \geq 2$ and $v_{k-1}$ has a prefix that belongs to $a^{l}(c|\bar{c}| d)$, which implies $l=k-1$. In either case, we get $k=l+1$.

Case 2. $w^{\prime} \in a^{k} c x \bar{c} d a^{l}(c|\bar{c}| \bar{d})$ for some $x \in H$. Then either $v_{k-1} \bar{c}$ is a prefix of $x \bar{c}$ or $x \bar{c}$ is a prefix of $v_{k-1} \bar{c}$. Since neither $v_{k-1}$ nor $x$ can start in $\bar{c}$, it follows that $v_{k-1}=\varepsilon$ if and only if $x=\varepsilon$. If $v_{k-1} \neq \varepsilon$ and $x \neq \varepsilon$, then either $v_{k-1}$ is a non-empty prefix of $x$ or $x$ is a non-empty prefix of $v_{k-1}$. Lemma 15 then implies $v_{k-1}=x$. So we always have $a^{k} c v_{k-1} \bar{c} d=a^{k} c x \bar{c} d$. By $(11)$, it follows that $v_{k-1} \bar{d}$ has a prefix that belongs to $a^{l}(c|\bar{c}| \bar{d})$. But the definition of $v_{n}$ implies that $v_{k-1} \bar{d}$ always has a prefix in $a^{k-1}(c \mid \bar{d})$. Therefore, $l=k-1$ and so $k=l+1$.

(ii). Exactly symmetric to part (i).

(iii). Suppose $u w v=\$ v_{n} \$$ and

$$
\begin{gathered}
w=w^{\prime} \bar{c} d w^{\prime \prime}, \\
w^{\prime} \in(c \mid \bar{d}) b^{k}, \quad w^{\prime \prime} \in a^{l}(c \mid \bar{d}) .
\end{gathered}
$$

By Lemma 17, part (iii), there exist $x, y \in H$ and $k^{\prime}, l^{\prime} \geq 1$ such that

$$
u w^{\prime} \in \$\left(\varepsilon \mid \hat{\Sigma}^{*}(c \mid d)\right) a^{k^{\prime}} c x, \quad w^{\prime \prime} v \in y \bar{d} b^{l^{\prime}}\left(\varepsilon \mid(\bar{c} \mid \bar{d}) \hat{\Sigma}^{*}\right) \$ .
$$

Since $x$ and $y$ are factors of $v_{n}$, Lemma 20 implies that $x=v_{i}$ and $y=v_{j}$ for some $i, j \geq 0$. If $i \geq 1$, then $v_{i}$ has $\bar{d} b^{i}$ as a suffix, so it follows that $k=i$. If $i=0$, then $u w^{\prime}$ ends in $c$, so $w^{\prime}=c$ and $k=0$. So we always have $k=i$. By a symmetric reasoning, we get $l=j$. It follows that

$$
u w v=u w^{\prime} \bar{c} d w^{\prime \prime} v \in \$\left(\varepsilon \mid \hat{\Sigma}^{*}(c \mid d)\right) a^{k^{\prime}} c v_{k} \bar{c} d v_{l} \bar{d} b^{l^{\prime}}\left(\varepsilon \mid(\bar{c} \mid \bar{d}) \hat{\Sigma}^{*}\right) \$ .
$$

Since $v_{k} \bar{c}$ has a prefix that belongs to $a^{k}(c \mid \bar{c})$ and $v_{l} \bar{d}$ has a prefix that belongs to $a^{l}(c \mid \bar{d})$, part (i) of this lemma implies $k^{\prime}=k+1=l+1$. Therefore, $k=l$.

We will make frequent use of Lemmas 18 and 21 in what follows. It will be important not to confuse part (i) and (ii) of Lemma 18 , on the one hand, and part (i) and (ii) of Lemma 21, on the other. The former state general properties of elements of $H$, while the latter express special properties of the strings $v_{n}$.

Lemma 22 Suppose $w \in \operatorname{fac}\left(\left\{v_{n} \mid n \in \mathbb{N}\right\}\right)$. 
(i) If $\psi(w) \in L$, then $w=a^{i} c v_{k} \bar{c}$ for some $i, k \geq 0$ such that $i \leq k+1$.

(ii) If $\psi(w) \in R$, then $w=d v_{k} \bar{d} b^{j}$ for some $j, k \geq 0$ such that $j \leq k+1$.

(iii) If $\psi(w) \in L R$, then $w=a^{i} c v_{k} \bar{c} d v_{k} \bar{d} b^{j}$ for some $i, j, k \geq 0$ such that $i, j \leq k+1$.

Proof (i). Suppose $u w v=v_{n}$ and $\psi(w) \in L=c V \bar{c}$. By Lemma 14 in the string $w, b$ cannot precede $a$ or $c$ and neither $a$ nor $b$ can follow $\bar{c}$. Hence $w=a^{i} c x \bar{c}$ for some $i \in \mathbb{N}$ and some $x$ such that $\psi(x) \in V$.

Since $u w v=u a^{i} c x \bar{c} v=v_{n} \in H$, Lemma 17 part (i), implies that there must be some $l \geq 1$ and $y \in H$ such that $l \geq i, a^{l}$ is a suffix of $u a^{i}$ and $a^{l} c x \bar{c} v \in y(\varepsilon \mid(\bar{c} \mid$ $\left.\bar{d}) \hat{\Sigma}^{*}\right)$. This means that $y$ must contain $a^{l} c$ as a prefix, so Lemma 20 implies $y=v_{l}=$ $a^{l} c v_{l-1} \bar{c} d v_{l-1} \bar{d} b^{l}$. Hence

$$
a^{l} c x \bar{c} v \in a^{l} c v_{l-1} \bar{c} d v_{l-1} \bar{d} b^{l}\left(\varepsilon \mid(\bar{c} \mid \bar{d}) \hat{\Sigma}^{*}\right) .
$$

This implies the following:

Either $x \bar{c}$ is a prefix of $v_{l-1} \bar{c}$, or else $v_{l-1} \bar{c}$ is a prefix of $x \bar{c}$.

We claim $x=v_{l-1}$. The desired conclusion follows from this by putting $k=l-1$.

Case 1. $l=1$. Then $v_{l-1}=v_{0}=\varepsilon$. Since $\psi(x) \in V$ implies that $x$ cannot start in $\bar{c}$, it is clear from (12) that $x$ must be $\varepsilon$. So the claim holds in this case.

Case 2. $l \geq 2$. It follows from 12 that either $\psi(x) \bar{c}$ is a prefix of $\psi\left(v_{l-1}\right) \bar{c}$ or vice versa. Since $l-1 \geq 1, \psi\left(v_{l-1}\right)$ starts in $c$. Then $\psi(x)$ must also start in $c$. Hence either $\psi(x)$ is a non-empty prefix of $\psi\left(v_{l-1}\right)$ or $\psi\left(v_{l-1}\right)$ is a non-empty prefix of $\psi(x)$. By Lemma 10 . we get $\psi\left(v_{l-1}\right)=\psi(x)$. Consequently, $x \bar{c}$ is not a prefix of $v_{l-1}$, and $v_{l-1} \bar{c}$ is not a prefix of $x$, so by (12), we can conclude $v_{l-1}=x$.

(ii). This is proved in an exactly symmetric way to (i).

(iii). By Part (i) and (ii) of this lemma, $w=a^{i} c v_{k} \bar{c} d v_{l} \bar{d} b^{j}$ for some $i, j, k \geq 0$ such that $i \leq k+1$ and $j \leq l+1$. Since $w$ contains a factor that belongs to $(c \mid \bar{d}) b^{\bar{k}} \bar{c} d a^{l}(c \mid$ $\bar{d})$, part (iii) of Lemma 21 gives $k=l$.

We now state and prove our main lemma. Let

$$
\begin{aligned}
\widehat{L} & =\left\{c v_{n} \bar{c} \mid n \in \mathbb{N}\right\}, \\
\widehat{R} & =\left\{d v_{n} \bar{d} \mid n \in \mathbb{N}\right\}, \\
\widehat{L R} & =\left\{c v_{n} \bar{c} d v_{n} \bar{d} \mid n \in \mathbb{N}\right\} .
\end{aligned}
$$

Then Lemma 22 implies

$$
\begin{gathered}
\psi^{-1}(L) \cap \operatorname{fac}\left(\left\{v_{n} \mid n \in \mathbb{N}\right\}\right) \subseteq a^{*} \widehat{L}, \\
\psi^{-1}(R) \cap \operatorname{fac}\left(\left\{v_{n} \mid n \in \mathbb{N}\right\}\right) \subseteq \widehat{R} b^{*}, \\
\psi^{-1}(L R) \cap \operatorname{fac}\left(\left\{v_{n} \mid n \in \mathbb{N}\right\}\right) \subseteq a^{*} \widehat{L R} b^{*} .
\end{gathered}
$$

Lemma 23 If $w \in \operatorname{fac}\left(\left\{v_{n} \mid n \in \mathbb{N}\right\}\right)$ and $w w \in \operatorname{fac}(H)$, then

$$
\psi(w) \in c^{*}\left|L d c^{*}\right| \bar{d}^{*}\left|\bar{d}^{*} \bar{c} R\right| V \bar{c} d c^{+} \mid \bar{d}^{+} \bar{c} d V .
$$


Proof Since $\varepsilon$ clearly belongs to the required set, assume $\psi(w) \in \Sigma^{+}$. Since $w w \in$ fac $(H)$ implies $\psi(w) \psi(w) \in \operatorname{fac}(V), \psi(w)$ must satisfy one of the five cases of Lemma 12 .

1. $\psi(w) \in(\bar{c} R \mid \bar{d})^{+}$.

2. $\psi(w) \in R(\bar{c} R \mid \bar{d})^{*} \bar{c}$.

3. $\psi(w) \in(c \mid L d)^{+}$.

4. $\psi(w) \in d(c \mid L d)^{*} L$.

5. $\psi(w) \in(V \mid R)(\bar{c} R \mid \bar{d})^{m} \bar{c} d(c \mid L d)^{n}(V \mid L)$ for some $m, n \geq 0$ such that $m \neq n$.

Below we treat the five cases in turn.

Case 1. $\psi(w) \in(\bar{c} R \mid \bar{d})^{+}$. We show that $\psi(w) \in \bar{d}^{+} \mid \bar{d}^{*} \bar{c} R$. Suppose by way of contradiction that $\psi(w) \in \bar{d}^{*} \bar{c} R(\bar{c} R \mid \bar{d})^{+}$. Lemma 14 says that in the string $w, a$ cannot precede $\bar{d}$ or $\bar{c}, b$ can follow only $\bar{d}$, and $\bar{d}$ can be followed only by $b$. Together with (14), this allows us to infer

$$
w \in b^{*}\left(\bar{d} b^{+}\right)^{*} \bar{c} \widehat{R} b^{+}\left((\bar{c} \widehat{R} \mid \bar{d}) b^{+}\right)^{*}(\bar{c} \widehat{R} \mid \bar{d}) b^{*} .
$$

Recall that $\widehat{R}$ consists of the strings $d v_{i} \bar{d}$. Recall also that $v_{i}=\varepsilon$ when $i=0$ and $v_{i}=a^{i} c v_{i-1} \bar{c} d v_{i-1} \bar{d} b^{i}$ otherwise. So if $w$ contains a factor that belongs to

$$
d v_{i} \bar{d} b^{j}(\bar{c} \mid \bar{d})
$$

then $w$ contains a factor that belongs to

$$
(d \mid \bar{d}) b^{i} \bar{d} b^{j}(\bar{c} \mid \bar{d})
$$

and part (ii) of Lemma 21 allows us to infer $j=i+1$. Hence $w$ must be of the form 8

$$
w=u x_{1} \ldots x_{m} \bar{c} d v_{k} \bar{d} b^{k+1} y_{1} \ldots y_{n} z
$$

where $m, n \geq 0$ and

$$
\begin{aligned}
u & \in b^{*}, \\
x_{i} & =\bar{d} b^{p_{i}} \quad \text { for some } p_{i} \geq 1, \\
y_{i} & \in\left(\bar{c} d v_{q_{i}} \bar{d} \mid \bar{d}\right) b^{q_{i}+1} \quad \text { for some } q_{i} \geq 0, \\
z & \in\left(\bar{c} d v_{l} \bar{d} \mid \bar{d}\right) b^{*} \quad \text { for some } l \geq 0 .
\end{aligned}
$$

Lemma 21, part (ii), also implies

$$
\begin{aligned}
q_{i+1} & =q_{i}+1 \quad \text { for } i=1, \ldots, n-1, \\
q_{1} & =k+1 \quad \text { if } n \geq 1 .
\end{aligned}
$$

So

$$
q_{i}=k+i \quad \text { for } i=1, \ldots, n .
$$

8 We will appeal to Lemma 21 similarly in Cases $2-5$ without explicitly going through this kind of reasoning. 
It immediately follows that

$$
\bar{d} b^{k+1} y_{1} \ldots y_{n} \text { contains } \bar{d} b^{k+n+1} \text { as a suffix. }
$$

Note that this holds even when $n=0$.

Next, we claim that

$$
d v_{k} \bar{d} b^{k+1} y_{1} \ldots y_{n} \text { has a suffix that belongs to } d\left(\bar{d} b^{*}\right)^{k+n+1} \text {. }
$$

By Lemma 19, this is clearly true when $n=0$. When $n \geq 1$, we can prove by induction on $i \in\{1, \ldots, n\}$ that $d v_{k} \bar{d} b^{k+1} y_{1} \ldots y_{i}$ always has a suffix in $d\left(\bar{d} b^{*}\right)^{k+i+1}$. For $i=0, d v_{k} \bar{d} b^{k+1}$ has a suffix in $d\left(\bar{d} b^{*}\right)^{k+1}$ by Lemma 19 . For $1 \leq i \leq n$, assume that $d v_{k} \bar{d} b^{k+1} y_{1} \ldots y_{i-1}$ has a suffix in $d\left(\bar{d} b^{*}\right)^{k+i}$. If $y_{i}=\bar{d} b^{q_{i}+1}=\bar{d} b^{k+i}$, then it follows that $d v_{k} \bar{d} b^{k+1} y_{1} \ldots y_{i}$ has a suffix in $d\left(\bar{d} b^{*}\right)^{k+i+1}$. If $y_{i}=\bar{c} d v_{q_{i}} \bar{d} b^{q_{i}+1}=$ $\bar{c} d v_{k+i} \bar{d} b^{k+i+1}$, then $y_{i}$ has a suffix in $d\left(\bar{d} b^{*}\right)^{k+i+1}$ by Lemma 19

Now note that

$$
w w \text { has a factor in } \bar{c} d v_{k} \bar{d} b^{k+1} y_{1} \ldots y_{n} z u x_{1} \ldots x_{m} \bar{c} d v_{k} \bar{d} b^{k+1}(\bar{c} \mid \bar{d}) \text {. }
$$

Since $w w \in \operatorname{fac}(H)$, this factor must also belong to fac $(H)$. We distinguish two cases.

Case 1.1. $z \in \bar{c} d v_{l} \bar{d} b^{*}$. Then by Lemma 19, $z u x_{1} \ldots x_{m}$ has a suffix in $d\left(\bar{d} b^{*}\right)^{l+1+m}$, so by Lemma 18 , part (ii), we get $l+1+m+1=k+1$, i.e.,

$$
k=l+m+1 .
$$

By (16), $w$ contains as a factor

$$
\bar{d} b^{k+n+1} z \in \bar{d} b^{k+n+1} \bar{c} d v_{l} \bar{d} b^{*}
$$

Since this factor belongs to $\operatorname{fac}\left(\left\{v_{n} \mid n \in \mathbb{N}\right\}\right)$, we must have

$$
l=k+n+1
$$

by Lemma 21, part (iii). But this last equation contradicts 19 .

Case 1.2. $z \in \bar{d} b^{*}$. By (17), we see that $d v_{k} \bar{d} b^{k+1} y_{1} \ldots y_{n} z u x_{1} \ldots x_{m}$ has a suffix in $d\left(\bar{d} b^{*}\right)^{k+n+1+1+m}=d\left(\bar{d} b^{*}\right)^{k+n+m+2}$. By Lemma 18, part (ii), we obtain from 18 that $k+n+m+2+1=k+1$, a contradiction.

We have derived a contradiction in each case. So the assumption that $\psi(w) \in$ $\bar{d}^{*} \bar{c} R(\bar{c} R \mid \bar{d})^{+}$is incorrect and $\psi(w)$ must be in $\bar{d}^{+} \mid \bar{d}^{*} \bar{c} R$. 
Case 2. $\psi(w) \in R(\bar{c} R \mid \bar{d})^{*} \bar{c}$. We derive a contradiction. By Lemma 14 in the string $w, \bar{c}$ can be followed only by $d$ and $\bar{d}$ can be followed only by $b$. Together with (14), this allows us to infer

$$
w \in \widehat{R} b^{+}\left((\bar{c} \widehat{R} \mid \bar{d}) b^{+}\right)^{*} \bar{c} .
$$

By Lemma 21, part (ii), $w$ must be of the form

$$
w=d v_{k} \bar{d} b^{k+1} y_{1} \ldots y_{n} \bar{c}
$$

where $n \geq 0$ and

$$
y_{i} \in\left(\bar{c} d v_{q_{i}} \bar{d} \mid \bar{d}\right) b^{q_{i}+1} \quad \text { for some } q_{i} \geq 0 .
$$

Lemma 21, part (ii), also implies

$$
\begin{aligned}
q_{i+1} & =q_{i}+1 \quad \text { for } i=1, \ldots, n-1, \\
q_{1} & =k+1 \quad \text { if } n \geq 1 .
\end{aligned}
$$

So we have

$$
q_{i}=k+i \quad \text { for } i=1, \ldots, n .
$$

As in Case 1, we can see that $v_{k} \bar{d} b^{k+1} y_{1} \ldots y_{n}$ has a suffix that belongs to $d\left(\bar{d} b^{*}\right)^{k+n+1}$. Since $w w$ has a factor in

$$
v_{k} \bar{d} b^{k+1} y_{1} \ldots y_{n} \bar{c} d v_{k} \bar{d} b^{k+1}(\bar{c} \mid \bar{d})
$$

and this factor belongs to fac $(H)$, Lemma 18 , part (ii), implies $k+n+1+1=k+1$, a contradiction.

Case 3. $\psi(w) \in(c \mid L d)^{+}$. This case is exactly symmetric to Case 1 and we can derive $\psi(w) \in c^{+} \mid L d c^{*}$.

Case 4. $\psi(w) \in d(c \mid L d)^{*} L$. This case is exactly symmetric to Case 2 and we can derive a contradiction.

Case 5. $\psi(w) \in(V \mid R)(\bar{c} R \mid \bar{d})^{m} \bar{c} d(c \mid L d)^{n}(V \mid L)$ for some $m, n \geq 0$ such that $m \neq n$. We show that $\psi(w) \in \bar{d}^{+} \bar{c} d V \mid V \bar{c} d c^{+}$. By Lemma $14 a$ cannot precede $\bar{c}$ or $\bar{d}$, and $b$ cannot follow $c$ or $d$. Together with (13), (14), and (15), this allows us to infer

$$
w \in\left(b^{*}\left|a^{*} \widehat{L R} b^{*}\right| \widehat{R} b^{*}\right)\left((\bar{c} \widehat{R} \mid \bar{d}) b^{*}\right)^{m} \bar{c} d\left(a^{*}(c \mid \widehat{L} d)\right)^{n}\left(a^{*}\left|a^{*} \widehat{L R} b^{*}\right| a^{*} \widehat{L}\right) .
$$

By Lemma 21, part (i) and (ii), we can write $w$ as

$$
w=x x_{1} \ldots x_{m} \bar{c} d y_{n} \ldots y_{1} y,
$$

where

$$
\begin{aligned}
& x \in b^{*}\left|a^{*} c v_{k} \bar{c} d v_{k} \bar{d} b^{k+1}\right| d v_{k} \bar{d} b^{k+1} \quad \text { for some } k \geq 0, \\
& y \in a^{*}\left|a^{l+1} c v_{l} \bar{c} d v_{l} \bar{d} b^{*}\right| a^{l+1} c v_{l} \bar{c} \quad \text { for some } l \geq 0, \\
& x_{i} \in\left(\bar{c} d v_{p_{i}} \bar{d} \mid \bar{d}\right) b^{p_{i}+1} \quad \text { for some } p_{i} \geq 0, \\
& y_{i} \in a^{q_{i}+1}\left(c \mid c v_{q_{i}} \bar{c} d\right) \quad \text { for some } q_{i} \geq 0 .
\end{aligned}
$$


Lemma 21, part (i) and (ii), also implies

$$
\begin{array}{ll}
p_{i+1}=p_{i}+1 & \text { for } i=1, \ldots, m-1, \\
q_{i+1}=q_{i}+1 & \text { for } i=1, \ldots, n-1 .
\end{array}
$$

We first show that

$$
y x=v_{j} \quad \text { for some } j .
$$

Since $w w$ contains $d y_{n} \ldots y_{1} y x x_{1} \ldots x_{m} \bar{c}$ as a factor and $w w \in \mathrm{fac}(H)$,

$$
(c \mid d) y x(\bar{c} \mid \bar{d}) \cap \mathrm{fac}(H) \neq \varnothing .
$$

By Lemma 14, the only symbol that can follow $\bar{c}$ in $y x$ is $d$ and the only symbol that can precede $d$ in $y x$ is $\bar{c}$. So $x=d v_{k} \bar{d} b^{k+1}$ if and only if $y=a^{l+1} c v_{l} \bar{c}$. Lemma 14 also implies that neither $a$ nor $c$ can follow $b$ or $\bar{d}$ in $y x$, so we cannot have both $x \in a^{*} c v_{k} \bar{c} d v_{k} \bar{d} b^{k+1}$ and $y \in a^{l+1} c v_{l} \bar{c} d v_{l} \bar{d} b^{*}$. Hence

$$
y x \in a^{*} b^{*}\left|a^{*} c v_{k} \bar{c} d v_{k} \bar{d} b^{k+1}\right| a^{l+1} c v_{l} \bar{c} d v_{l} \bar{d} b^{*} \mid a^{l+1} c v_{l} \bar{c} d v_{k} \bar{d} b^{k+1} .
$$

If $y x \in a^{*} b^{*}$, Lemma 14 together with (23) implies $y x=\varepsilon=v_{0}$. Otherwise, Lemmas 18 and 19 together with 23 imply

$$
y x=a^{j+1} c v_{j} \bar{c} d v_{j} \bar{d} b^{j+1}=v_{j+1},
$$

where $j=k$ or $j=l$. This establishes 22].

Since $m \neq n$, either $m \geq 1$ or $n \geq 1$. We distinguish three cases:

Case 5.1. $m \geq 1, n \geq 1$. In this case, $w w$ contains a factor in

$$
(c \mid d) y_{1} v_{j} x_{1}(\bar{c} \mid \bar{d})
$$

This factor is in $\operatorname{fac}(H)$. Since $\psi(w w) \in \operatorname{fac}(V) \subseteq \operatorname{fac}\left(D_{2}^{*}\right)$, we have $\psi\left(y_{1} v_{j} x_{1}\right) \in$ $\operatorname{fac}\left(D_{2}^{*}\right)$. By Lemma $4, \operatorname{nf}\left(\psi\left(y_{1} v_{j} x_{1}\right)\right) \in(\bar{c} \mid \bar{d})^{*}(c \mid d)^{*}$, and it follows that

$$
y_{1} v_{j} x_{1} \in a^{q_{1}+1} c v_{j} \bar{c} d v_{p_{1}} \bar{d} b^{p_{1}+1} \mid a^{q_{1}+1} c v_{q_{1}} \bar{c} d v_{j} \bar{d} b^{p_{1}+1} .
$$

So

$$
(c \mid d)\left(a^{q_{1}+1} c v_{j} \bar{c} d v_{p_{1}} \bar{d} b^{p_{1}+1} \mid a^{q_{1}+1} c v_{q_{1}} \bar{c} d v_{j} \bar{d} b^{p_{1}+1}\right)(\bar{c} \mid \bar{d}) \cap \operatorname{fac}(H) \neq \varnothing .
$$

By Lemmas 18 and 19 , we obtain $p_{1}=q_{1}=j$. By 201 and $\left[21\right.$, then, we get $p_{m}=$ $j+m-1$ and $q_{n}=j+n-1$. Since

$$
x_{m} \bar{c} d y_{n} \in\left(\bar{c} d v_{j+m-1} \bar{d} \mid \bar{d}\right) b^{j+m} \bar{c} d a^{j+n}\left(c \mid c v_{j+n-1} \bar{c} d\right)
$$

is a factor of $w$, we get $j+m=j+n$ by Lemma 21, part (iii), but this contradicts $m \neq n$. 
Case 5.2. $m \geq 1, n=0$. Since

$$
w w=x x_{1} \ldots x_{m} \bar{c} d v_{j} x_{1} \ldots x_{m} \bar{c} d y
$$

and $\psi(w w) \in \operatorname{fac}(V) \subseteq \operatorname{fac}\left(D_{2}^{*}\right)$, we get $\psi\left(d v_{j} x_{1}\right) \in \operatorname{fac}\left(D_{2}^{*}\right)$. By Lemma 4 $\operatorname{nf}\left(\psi\left(d v_{j} x_{1}\right)\right)=\operatorname{nf}\left(d \psi\left(x_{1}\right)\right) \in(\bar{c} \mid \bar{d})^{*}(c \mid d)^{*}$. Hence we must have

$$
x_{1}=\bar{d} b^{p_{1}+1} .
$$

By 20$], p_{i}=p_{1}+i-1$ for $i=1, \ldots, m$. We consider three subcases, depending on whether $x \in b^{*}$, and whether $x_{i}=\bar{d} b^{p_{1}+i}$ for all $i=1, \ldots, m$.

Case 5.2.1. $x \in b^{*}$ and $x_{i}=\bar{d} b^{p_{1}+i}$ for all $i=1, \ldots, m$. Then since $y x=v_{j}$, either $x=y=\varepsilon$ or $j=l+1$ and $y \in a^{l+1} c v_{l} \bar{c} d v_{l} \bar{d} b^{*}$. Hence

$$
\psi(w) \in \bar{d}^{+} \bar{c} d V
$$

Case 5.2.2. $x \notin b^{*}$ and $x_{i}=\bar{d} b^{p_{1}+i}$ for all $i=1, \ldots, m$. Then $j=k+1, y x=v_{k+1}$, and $d v_{k} \bar{d} b^{k+1}$ is a suffix of $x$. Since $w$ contains a factor in

$$
d v_{k} \bar{d} b^{k+1} x_{1}(\bar{c} \mid \bar{d})=d v_{k} \bar{d} b^{k+1} \bar{d} b^{p_{1}+1}(\bar{c} \mid \bar{d}),
$$

we get $p_{1}=k+1$ by Lemma 21 part (ii). By Lemma 19 we also see that $x x_{1} \ldots x_{m}$ has a suffix in $d\left(\bar{d} b^{*}\right)^{k+m+1}$. Since $w w$ has a factor in

$$
\begin{aligned}
x x_{1} \ldots x_{m} \bar{c} d v_{k+1} x_{1}(\bar{c} \mid \bar{d}) & =x x_{1} \ldots x_{m} \bar{c} d v_{k+1} \bar{d} b^{k+2}(\bar{c} \mid \bar{d}) \\
& \subseteq \hat{\Sigma}^{*} d\left(\bar{d} b^{*}\right)^{k+m+1} \bar{c} d H \bar{d} b^{k+2}(\bar{c} \mid \bar{d}),
\end{aligned}
$$

we get by Lemma 18 , part (ii),

$$
k+m+1+1=k+2,
$$

which contradicts $m \geq 1$.

Case 5.2.3. $x_{h}=\bar{c} d v_{p_{1}+h-1} \bar{d} b^{p_{1}+h}$ for some $h \in\{2, \ldots, m\}$. (Recall $x_{1}=\bar{d} b^{p_{1}+1}$.) We can assume $h$ to be the largest such number, i.e., $x_{i}=\bar{d} b^{p_{1}+i}$ for all $i \in\{h+1, \ldots, m\}$. By Lemma 19, $x_{h}$ has a suffix in $d\left(\bar{d} b^{*}\right)^{p_{1}+h}$. It follows that $x_{h} \ldots x_{m}$ has a suffix in $d\left(\bar{d} b^{*}\right)^{p_{1}+m}$. Since $w w$ has a factor in

$$
\begin{aligned}
x_{h} \ldots x_{m} \bar{c} d v_{j} x_{1}(\bar{c} \mid \bar{d}) & =x_{h} \ldots x_{m} \bar{c} d v_{j} \bar{d} b^{p_{1}+1}(\bar{c} \mid \bar{d}) \\
& \subseteq \hat{\Sigma}^{*} d\left(\bar{d} b^{*}\right)^{p_{1}+m} \bar{c} d H \bar{d} b^{p_{1}+1}(\bar{c} \mid \bar{d}),
\end{aligned}
$$

we get by Lemma 18 , part (ii),

$$
p_{1}+m+1=p_{1}+1
$$

which contradicts $m \geq 1$.

Case 5.3. $m=0, n \geq 1$. This case is exactly symmetric to the preceding case, and we can conclude

$$
\psi(w) \in V \bar{c} d c^{+} .
$$

This concludes the proof of the lemma. 
Theorem 24 For each $n \geq 0$, the string $v_{n}$ is almost anti-iterative in $H$.

Before embarking on the proof of the theorem, let us consider a simple example:

$$
v_{2}=\underbrace{a a c}_{w_{1}} \underbrace{a c \bar{c} d \bar{d}}_{u_{1}} \underbrace{b \bar{c} d a c \bar{c} d \bar{d} b \bar{d} b}_{w_{2}} \underbrace{b}_{w_{3}} .
$$

In this example, $u_{0}=u_{2}=u_{3}=\varepsilon$. Note

$$
\psi\left(w_{1}\right)=c, \quad \psi\left(w_{2}\right) \in \bar{c} R, \quad \psi\left(w_{3}\right)=\varepsilon .
$$

We have

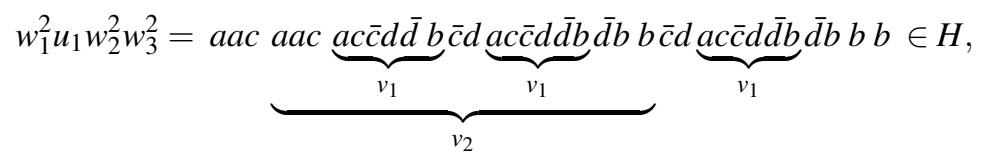

but

$$
\begin{aligned}
& w_{1}^{3} u_{1} w_{2}^{3} w_{3}^{3}= \\
& \text { aac aac aac } \underbrace{\underbrace{a c \bar{c} d \bar{d} b}_{v_{1}} \bar{c} d \underbrace{a c \bar{c} d \bar{d} b}_{v_{1}} \bar{d} b b}_{v_{2}} \bar{c} d \underbrace{a c \bar{c} d \bar{d} b}_{v_{1}} \bar{d} b b \bar{c} d \underbrace{a c \bar{c} d \bar{d} b}_{v_{1}} \bar{d} b b b b \notin H
\end{aligned}
$$

After the occurrence of $\bar{d}$ following the third occurrence of $v_{1}$, one should find $b^{3}$, rather than $b^{2}$, in order to have a string in $H$ (as required by Lemma 18 , part (ii)).

Proof (of Theorem 24) Suppose that $v_{n}=u_{0} w_{1} u_{1} \ldots w_{k} u_{k}$ and $w_{1} \ldots w_{k} \neq \varepsilon$. If there is some $j$ such that $w_{j}^{3}$ is not in $\operatorname{fac}(H)$, then there is no $i \geq 3$ such that $u_{0} w_{1}^{i} u_{1} \ldots w_{k}^{i} u_{k} \in$ $H$, and the conclusion of the theorem is clearly satisfied. Hence we may assume that each $w_{j}^{3}$ belongs to $\operatorname{fac}(H)$.

Suppose that $u_{0} w_{1}^{h} \ldots w_{k}^{h} u_{k} \in H$ for some $h>1$. We show that such $h$ is unique.

Since $w_{j}^{2}$ is a factor of $w_{j}^{3}$ and hence belongs to fac $(H)$, by Lemma 23 each $\psi\left(w_{j}\right)$ must belong to one of the six sets

$$
c^{*}, \quad L d c^{*}, \quad \bar{d}^{*}, \quad \bar{d}^{*} \bar{c} R, \quad V \bar{c} d c^{+}, \quad \bar{d}^{+} \bar{c} d V .
$$

Since $w_{1} \ldots w_{k} \neq \varepsilon$, we have $u_{0} w_{1} u_{1} \ldots w_{k} u_{k} \neq u_{0} w_{1}^{h} u_{1} \ldots w_{k}^{h} u_{k}$. By Lemma 13 , we know that $\psi\left(u_{0} w_{1} u_{1} \ldots w_{k} u_{k}\right) \neq \psi\left(u_{0} w_{1}^{h} u_{1} \ldots w_{k}^{h} u_{k}\right)$. Therefore, it cannot be that $\psi\left(w_{j}\right)=\varepsilon$ for all $j$. Since both $\psi\left(u_{0} w_{1} u_{1} \ldots w_{k} u_{k}\right)$ and $\psi\left(u_{0} w_{1}^{h} u_{1} \ldots w_{k}^{h} u_{k}\right)$ belong to $V$, the string $\psi\left(w_{1}\right) \ldots \psi\left(w_{k}\right)$ must have the same number of occurrences of $c, \bar{c}, \bar{d}, \bar{d}$. It follows that there is a $j$ such that $\psi\left(w_{j}\right) \in L d c^{*}\left|\bar{d}^{*} \bar{c} R\right| V \bar{c} d c^{+} \mid \bar{d}^{+} \bar{c} d V$. 
Case 1. $\psi\left(w_{j}\right) \in L d c^{*}$. Lemma 14 implies that in the string $w_{j}, b$ can follow only $\bar{d}$. So

$$
w_{j} \in v d\left(a^{*} c\right)^{*} a^{*}
$$

for some $v \in \operatorname{fac}\left(\left\{v_{n} \mid n \in \mathbb{N}\right\}\right)$ such that $\psi(v) \in L$. By Lemma 22, $v \in a^{*} c v_{l} \bar{c}$ for some $l \geq 0$. Lemma 14 also implies that in $u_{0} w_{1} u_{1} \ldots w_{k} u_{k}$, (i) the only symbols that can precede $a$ are $a, c$, and $d$, (ii) the only symbols that can follow $a$ are $a$ and $c$, and (iii) the only symbols that can follow $c$ or $d$ are $a, \bar{c}$, and $\bar{d}$. Hence we can write

$$
\begin{aligned}
u_{0} w_{1} u_{1} \ldots w_{j-1} u_{j-1} & \in\left(\varepsilon \mid \hat{\Sigma}^{*}(c \mid d)\right) a^{m_{0}}, \\
w_{j} & \in a^{m_{1}} c v_{l} \bar{c} d\left(a^{*} c\right)^{p} a^{m_{2}}, \\
u_{j} w_{j+1} u_{j+1} \ldots w_{k} u_{k} & \in\left(a^{*} c\right)^{q}(\bar{c} \mid \bar{d}) \hat{\Sigma}^{*},
\end{aligned}
$$

for some $l, m_{0}, m_{1}, m_{2}, p, q \geq 0$. We get $m_{0}+m_{1}=l+1$ by Lemma 21, part (i), and $m_{0}+m_{1}=p+q+1$ by Lemma 18 , part (i). Hence $l=p+q$.

Let $g \geq j$ the largest number such that $u_{j} w_{j+1} \ldots u_{g-1} w_{g} \in\left(a^{*} c\right)^{*} a^{*}$. Let $r$ be the number of occurrences of $c$ in $w_{j+1} \ldots w_{g}$. Then for every $i \geq 1$,

$$
u_{j} w_{j+1}^{i} u_{j+1} \ldots w_{k}^{i} u_{k} \in\left(a^{*} c\right)^{q+(i-1) r}(\bar{c} \mid \bar{d}) \hat{\Sigma}^{*} .
$$

Thus, $w_{j}^{h} u_{j} w_{j+1}^{h} u_{j+1} \ldots w_{k}^{h} u_{k}$ has a factor in

$$
d\left(a^{*} c\right)^{p} a^{m_{2}+m_{1}} c v_{l} \bar{c} d\left(a^{*} c\right)^{p} a^{m_{2}}\left(a^{*} c\right)^{q+(h-1) r}(\bar{c} \mid \bar{d}) .
$$

Since this factor is in $\operatorname{fac}(H)$, Lemma 18 , part (i), implies

$$
\begin{aligned}
m_{2}+m_{1} & =p+q+(h-1) r+1 \\
& =(h-1) r+l+1 .
\end{aligned}
$$

Note that the string $w_{j}^{3}$ has a factor in

$$
d\left(a^{*} c\right)^{p} a^{m_{2}+m_{1}} c v_{l} \bar{c} d\left(a^{*} c\right)^{p} a^{m_{2}+m_{1}} c v_{l} \bar{c} .
$$

Since we assumed that $w_{j}^{3} \in \operatorname{fac}(H)$, this factor is also in $\operatorname{fac}(H)$. By Lemma $19, v_{l} \bar{c}$ has a prefix that belongs to $\left(a^{*} c\right)^{l} \bar{c}$. By Lemma 18 , part (i), then, we have

$$
\begin{aligned}
m_{2}+m_{1} & =p+1+l+1 \\
& =p+l+2 .
\end{aligned}
$$

From (24) and (25), we get

$$
(h-1) r=p+1 .
$$

Since $p \geq 0$, this implies $r \neq 0$ and

$$
h=\frac{p+1}{r}+1,
$$

which shows that $h$ is unique.

Case 2. $\psi\left(w_{j}\right) \in \bar{d}^{*} \bar{c} R$. This case is exactly symmetric to the preceding case. 
Case 3. $\psi\left(w_{j}\right) \in V \bar{c} d c^{+}$. We can use Lemma 14 to infer

$$
\begin{aligned}
w_{j} & \in v \bar{c} d\left(a^{*} c\right)^{+} a^{*}, \\
u_{j} w_{j+1} u_{j+1} \ldots w_{k} u_{k} & \in\left(a^{*} c\right)^{*} \bar{c} \hat{\Sigma}^{*}
\end{aligned}
$$

for some string $v \in \operatorname{fac}\left(\left\{v_{n} \mid n \in \mathbb{N}\right\}\right)$ such that $\psi(v) \in V$. By Lemma21, part (i), we can write

$$
\begin{aligned}
w_{j} & \in v \bar{c} d a^{l_{1}+l_{2}} c \ldots a^{l_{1}+1} c a^{m_{1}}, \\
u_{j} w_{j+1} u_{j+1} \ldots w_{k} u_{k} & \in a^{m_{2}} c a^{l_{1}-1} c \ldots c a^{1} c \bar{c} \hat{\Sigma}^{*} \\
& \subseteq\left(a^{*} c\right)^{l_{1}} \bar{c} \hat{\Sigma}^{*} .
\end{aligned}
$$

for some $l_{1}, m_{1}, m_{2} \geq 0$ and $l_{2} \geq 1$ such that $m_{1}+m_{2}=l_{1}$. Similarly to Case 1 , there must be some $r \geq 0$ such that

$$
u_{j} w_{j+1}^{i} u_{j+1} \ldots w_{k}^{i} u_{k} \in\left(a^{*} c\right)^{l_{1}+(i-1) r} \bar{c} \hat{\Sigma}^{*}
$$

for all $i \geq 1$. Then $w_{j}^{h} u_{j} w_{j+1}^{h} u_{j+1} \ldots w_{k}^{h} u_{k}$ has a factor in

$$
\begin{gathered}
(c \mid d) a^{l_{1}+1} c a^{m_{1}} v \bar{c} d a^{l_{1}+l_{2}} c \ldots a^{l_{1}+1} c a^{m_{1}}\left(a^{*} c\right)^{l_{1}+(h-1) r} \bar{c} \hat{\Sigma}^{*} \\
\subseteq(c \mid d) a^{l_{1}+1} c a^{m_{1}} v \bar{c} d\left(a^{*} c\right)^{l_{2}+l_{1}+(h-1) r} \bar{c} \hat{\Sigma}^{*} .
\end{gathered}
$$

This factor is in $\operatorname{fac}(H)$. Note that the above inclusion holds even when $l_{1}=r=0$, since $l_{1}=0$ implies $m_{1}=0$.

We show that $a^{m_{1}} v \in H$. Recall $\psi(v) \in V$ and $v \in$ fac $\left(\left\{v_{n} \mid n \in \mathbb{N}\right\}\right)$. If $\psi(v)=\varepsilon$, then $v \in(a \mid b)^{*}$, but since $c a^{m_{1}} v \bar{c} \in$ fac $(H)$, Lemma 14 implies $a^{m_{1}} v=\varepsilon \in H$. If $\psi(v) \in L R$, Lemma 22 implies that $a^{m_{1}} v \in a^{*} c v_{l} \bar{c} d v_{l} \bar{d} b^{*}$ for some $l$. Since $c a^{m_{1}} v \bar{c} \in$ fac $(H)$, it follows from Lemma 19 and Lemma 18 part (i) and (ii), that $a^{m_{1}} v=a^{l+1} c v_{l} \bar{c} d v_{l} \bar{d} b^{l+1}=v_{l+1} \in H$.

So the set (26) is included in

$$
(c \mid d) a^{l_{1}+1} c H \bar{c} d\left(a^{*} c\right)^{l_{2}+l_{1}+(h-1) r} \bar{c} \hat{\Sigma}^{*} .
$$

Since there is an element of $\operatorname{fac}(H)$ belonging to this set, we obtain by Lemma 18 part (i)

$$
l_{1}+1=l_{2}+l_{1}+(h-1) r+1 .
$$

Since $h>1, r \geq 0$ and $l_{2} \geq 1$, this is a contradiction.

Case 4. $\psi\left(w_{j}\right) \in \bar{d}^{+} \bar{c} d V$. This case is exactly symmetric to the preceding case.

Corollary 25 The language $H$ is not iterative.

Corollary 26 There is a 3-MCFL that is not $k$-iterative for any $k$. 


\section{Conclusion}

We have proved that the language $H$ is a 3-MCFL that is not iterative. A simple consequence of this theorem is that if $\mathscr{C}$ is a subclass of the class MCFL of multiple context-free languages and $\mathscr{C}$ consists entirely of iterative sets, then the language $H$ does not belong to $\mathscr{C}$ and hence $\mathscr{C}$ must be a proper subclass of MCFL.

Kanazawa and Salvati [8] showed that the class $\mathrm{MCFL}_{\mathrm{wn}}$ of well-nested multiple context-free languages is properly included in MCFL, and in particular, the language $\left\{w \# w \mid w \in D_{2}^{*}\right\}$ belongs to MCFL - MCFL ${ }_{w n}$. Since every language in $\mathrm{MCFL}_{\mathrm{wn}}$ is $k$-iterative for some $k$, the language $H$ serves as a further witness to the separation of MCFL and $\mathrm{MCFL}_{\mathrm{wn}}$.

Another subclass of MCFL that only contains languages that are $k$-iterative for some $k$ is the class of languages in Weir's control language hierarchy [16, 12,7]. As far as we know, it has been an open question whether the inclusion of the control language hierarchy in the class of multiple context-free languages is proper. The language $H$ serves as a witness to the properness of the inclusion.

Corollary 27 There is a 3-MCFL that does not belong to Weir's control language hierarchy.

\section{References}

1. Berstel, J., Boasson, L.: Context-free languages. In: J. van Leeuwen (ed.) Handbook of Theoretical Computer Science, vol. B, pp. 59-102. Elsevier, Amsterdam (1990)

2. Greibach, S.A.: Hierarchy theorems for two-way finite state transducers. Acta Informatica 11, 89-101 (1978)

3. Greibach, S.A.: One-way finite visit automata. Theoretical Computer Science 6, 175-221 (1978)

4. Groenink, A.V.: Mild context-sensitivity and tuple-based generalizations of context-free grammar. Linguistics and Philosophy 20(6), 607-636 (1997)

5. Groenink, A.V.: Surface without Structure. Ph.D. thesis, University of Utrecht (1997)

6. Kanazawa, M.: The pumping lemma for well-nested multiple context-free languages. In: V. Diekert, D. Nowotka (eds.) Developments in Language Theory: 13th International Conference, DLT 2009, Lecture Notes in Computer Science, vol. 5583, pp. 312-325. Springer, Berlin (2009)

7. Kanazawa, M., Salvati, S.: Generating control languages with abstract categorial grammars. In: Preliminary Proceedings of FG-2007: The 12th Conference on Formal Grammar (2007)

8. Kanazawa, M., Salvati, S.: The copying power of well-nested multiple context-free grammars. In A.H. Dediu, H. Fernau, C. Martín-Vide (eds.) Language and Automata Theory and Applications, Fourth International Conference, LATA 2010, Lecture Notes in Computer Science, vol. 6031, pp. 344-355. Springer, Berlin (2010)

9. Kasami, T., Seki, H., Fujii, M.: Generalized context-free grammars, multiple context-free grammars and head grammars. Tech. rep., Osaka University (1987)

10. Kracht, M.: The Mathematics of Language. Mouton de Gruyter, Berlin (2003)

11. Michaelis, J.: On Formal Properties of Minimalist Grammars. Linguistics in Potsdam 13. Universitätsbibliothek, Publikationsstelle, Potsdam. Ph.D. thesis, ISBN 3-935024-28-2

12. Palis, M.A., Shende, S.M.: Pumping lemmas for the control language hierarchy. Mathematical Systems Theory 28(3), 199-213 (1995)

13. Radzinski, D.: Chinese number-names, tree adjoining languages, and mild context-sensitivity. Computational Linguistics 17(3), 277-299 (1991)

14. Seki, H., Matsumura, T., Fujii, M., Kasami, T.: On multiple context-free grammars. Theoretical Computer Science 88(2), 191-229 (1991)

15. Vijay-Shanker, K., Weir, D.J., Joshi, A.K.: Characterizing structural descriptions produced by various grammatical formalisms. In: 25th Annual Meeting of the Association for Computational Linguistics, pp. 104-111 (1987) 
16. Weir, D.J.: A geometric hierarchy beyond context-free languages. Theoretical Computer Science 104(2), 235-261 (1992). DOI 10.1016/0304-3975(92)90124-X 